\title{
Peroxiredoxin-2 Protects against 6-Hydroxydopamine-Induced Dopaminergic Neurodegeneration via Attenuation of the Apoptosis Signal-Regulating Kinase (ASK1) Signaling Cascade
}

\author{
Xiaoming Hu, ${ }^{1,2 \star}$ Zhongfang Weng, ${ }^{1 *}$ Charleen T. Chu, ${ }^{1,3}$ Lili Zhang, ${ }^{1}$ Guodong Cao, ${ }^{1,2}$ Yanqin Gao, ${ }^{1,2}$ \\ Armando Signore, ${ }^{1}$ Jianhui Zhu, ${ }^{3}$ Teresa Hastings, ${ }^{1}$ J. Timothy Greenamyre, ${ }^{1}$ and Jun Chen ${ }^{1,2}$ \\ ${ }^{1}$ Pittsburgh Institute of Neurodegenerative Diseases, University of Pittsburgh School of Medicine, Pittsburgh, Pennsylvania 15213, 2State Key Laboratory of \\ Medical Neurobiology and Institute of Brain Science, Fudan University, Shanghai 200032, China, and 3Department of Pathology, University of Pittsburgh \\ School of Medicine, Pittsburgh, Pennsylvania 15261
}

The peroxiredoxin (PRX) family of antioxidant enzymes helps maintain the intracellular reducing milieu and suppresses apoptosis in non-neuronal cells. However, whether PRX can inhibit neuronal apoptosis through specific signaling mechanisms remains poorly understood. Induction of PRX2, the most abundant neuronal PRX, occurs in Parkinson's disease (PD) patient brains, but its functional impact is unclear. In the present study, we used the dopaminergic (DA) toxin 6-hydroxydopamine (6-OHDA) to model PD and explore the protective effect and mechanisms of PRX on DA neurons. Of the 2-cysteine PRXs that were tested in MN9D DA neurons, endogenous PRX2 was most beneficial to cell survival. Lentivirus-mediated PRX2 overexpression conferred marked in vitro and in vivo neuroprotection against 6-OHDA toxicity in DA neurons, and preserved motor functions involving the dopamine system in mouse. In addition to its role as an antioxidant enzyme, PRX2 exhibited anti-apoptotic effects in DA neurons via suppression of apoptosis signal-regulating kinase (ASK1)-dependent activation of the c-Jun N-terminal kinase/c-Jun and p38 pro-death pathways, which are also activated in DA neurons of postmortem PD brains. PRX2 inhibited 6-OHDA-induced ASK1 activation by modulating the redox status of the endogenous ASK1 inhibitor thioredoxin (Trx). PRX2 overexpression maintained Trx in a reduced state by inhibiting the cysteine thiol-disulfide exchange, thereby preventing its dissociation from ASK1. This study describes a previously undefined mechanism by which redox-sensitive molecules signal via apoptotic pathways in response to PD-relevant toxic stress in DA neurons. Our results also suggest that PRX2 and ASK1 may be potential targets for neuroprotective intervention in PD.

\section{Introduction}

Dopaminergic (DA) neurodegeneration in the substantia nigra pars compacta $(\mathrm{SNc})$ is a hallmark of idiopathic Parkinson's disease (PD). Although the mechanism underlying the selective neuronal loss in $\mathrm{SNc}$ remains elusive, oxidative stress is strongly implicated as a contributing factor (Palacino et al., 2004; Przedborski and Ischiropoulos, 2005; Tsang and Chung, 2009). It has been demonstrated that increased reactive oxygen species (ROS) activates the mitochondrial apoptotic pathways in DA neurons in experimental models of PD (Choi et al., 2004; Saito et al., 2007).

Received Aug. 27, 2010; revised Sept. 30, 2010; accepted 0ct. 23, 2010.

This project was supported by National Institutes of Health Grants NS062157, NS056118, and NS036736 (to J.C.), NS059806 (to J.T.G., C.C., G.C., T.H., and J.C.), and AG026389 (to C.C.); American Heart Association Grant 10POST4150028 (to X.H.); and Chinese National Science Foundation Grant 30870794 (to Y.G.). We thank Hui Gao for performing HPLC measurement of catecholamine levels, Jason Callio for performing paraffin immunohistochemistry, and Carol Culver for editorial assistance.

*X.H. and Z.W. contributed equally to this work.

Correspondence should be addressed to Dr. Jun Chen, Department of Neurology, S-507, Biomedical Science Tower, University of Pittsburgh School of Medicine, Pittsburgh, PA 15213. E-mail: chenj2@upmc.edu. DOI:10.1523/JNEUROSCI.4589-10.2011

Copyright $\odot 2011$ the authors $\quad 0270-6474 / 11 / 310247-15 \$ 15.00 / 0$
To counter this oxidative stress, neurons mobilize a complex ROS-scavenging network.

Peroxiredoxins (PRXs) are one of the ROS management systems that maintain the intracellular reducing milieu via redox reactions at certain cysteine residues. They are divided into the following three classes: typical 2-Cys PRXs (PRX1-4); atypical 2-Cys PRXs (PRX5); and 1-Cys PRXs (PRX6). The role of neuronal-expressed PRXs, particularly PRX2 in PD, is just beginning to be understood. PRX2 levels are significantly elevated in SNc specimens from PD patients (Basso et al., 2004). Nitrosylation (Fang et al., 2007) and phosphorylation (Qu et al., 2007) of PRX2, modifications that inhibit PRX2 enzyme activity and its protective effect against oxidative stress, are also observed in human PD brains and in PD models. Recent evidence suggests that the 2-Cys PRXs not only control the levels of intracellular peroxide but directly participate in apoptosis signaling cascades. For example, PRX2 can hinder the initiation of apoptosis: enhanced expression of PRX2 in tumor cells inhibits apoptosis induced by $\mathrm{H}_{2} \mathrm{O}_{2}$, cisplatin, or radiation, thereby rendering tumor cells resistant to oxidative stress-based therapies (Park et al., 2000; Chung et al., 2001; Bae et al., 2007). Overexpression of PRX2 signifi- 
Table 1. Sequence of shRNA against murine PRXs or ASK1

\begin{tabular}{|c|c|}
\hline Gene & shRNA sequence \\
\hline \multirow[t]{2}{*}{ PRX1 } & Targeting sequence 1: 5' -GGATTCTCACTTCTGTCATCT-3' \\
\hline & Targeting sequence $2: 5^{\prime}$-GCGCACCATTGCTCAGGATTA- $3^{\prime}$ \\
\hline \multirow[t]{2}{*}{ PRX2 } & Targeting sequence $1: 5^{\prime}$ - GGATGGTGCCTTCAAGGAAAT-3' \\
\hline & Targeting sequence $2: 5^{\prime}$ - GCAAGGAATACTTCTCCAAAC-3' \\
\hline \multirow[t]{2}{*}{$P R X 3$} & Targeting sequence 1: 5' - GAGCTGAGTCTCGACGACTTT-3' \\
\hline & Targeting sequence $2: 5^{\prime}$ - GTAGTTGCAGTTTCAGTGGAT-3' \\
\hline \multirow[t]{2}{*}{ PRX4 } & Targeting sequence 1: 5' - GCAAAGCCAAGATCTCCAAGC-3' \\
\hline & Targeting sequence $2: 5^{\prime}$ - GCTCAAACTGACTGACTATCG-3' \\
\hline \multirow[t]{2}{*}{ ASK1 } & Targeting sequence $1: 5^{\prime}$-CCAACAACATCATCCTCTA-3' \\
\hline & Targeting sequence $2: 5^{\prime}$-TGAACACCATTACCGAAGA-3' \\
\hline
\end{tabular}

cantly protects cardiomyocytes from apoptosis induced by ischemia/reperfusion or $\mathrm{H}_{2} \mathrm{O}_{2}$ ( $\mathrm{Zhao}$ et al., 2009). Despite the evidence for the role of PRX2 in preventing apoptosis, the role of PRXs in regulating neuronal cell death associated with neurological diseases has not been thoroughly evaluated.

Activation of the c-Jun N-terminal kinase (JNK) and p38 mitogen-activated protein kinase (MAPK), two regulatory signaling pathways upstream of mitochondrial apoptosis, has been observed in PD models and appears to be critical in mediating DA cell death (Choi et al., 2004; Klintworth et al., 2007). While the precise upstream mechanism leading to JNK and p38 activation in DA neurons is not known, apoptosis signal-regulating kinase (ASK1) is emerging as a potential key player (Ouyang and Shen, 2006). The activity of both 2-Cys PRXs and ASK1 can be regulated by thioredoxin (Trx) in an oxidative state-dependent manner (Rhee et al., 2005; Fujino et al., 2007), suggesting a linkage of these molecules in oxidative stress-associated cell death signaling.

In this study, we report opposite functional roles of PRX2 and ASK1 in DA neuronal death in the 6-hydroxydopamine (6OHDA) model of PD. We present both in vitro and in vivo evidence that PRX2 confers remarkable protection against 6-OHDA-induced DA neuronal loss via suppression of ASK1dependent activation of JNK/c-Jun and p38 pro-death signaling pathways. Furthermore, we demonstrate that PRX2 negatively regulates ASK1 activation by modulating the redox status of Trx and inhibiting the dissociation of Trx from ASK1.

\section{Materials and Methods}

Cell culture and neurotoxin exposure. The MN9D DA neuronal cell line was maintained in DMEM (Invitrogen) supplemented with $10 \%$ fetal calf serum. Neuronal differentiation was induced using $1 \mathrm{~mm} n$-butyrate for $5 \mathrm{~d}$ before cells were used for experiments. The neurotoxin 6 -OHDA.HBr (Sigma) was made fresh for each experiment in a nonoxidizing vehicle (Ding et al., 2004). Cultures were washed with serum-free media before exposure to 50 or $100 \mu \mathrm{M} 6$-OHDA for $30 \mathrm{~min}$, followed by two washes of serum-free media, and fed with serum-containing media until they were harvested for various assays.

Construction of viral vectors. To construct lentiviral vectors (Lenti) overexpressing human full-length PRX2 or its dominant-negative form (Lenti-PRX2dn), human full-length ASK1 (Lenti-ASK1 h) or its dominant-negative form (Lenti-ASK1dn, containing the K709R mutation), PRX1, PRX3, and PRX4, the hemagglutinin (HA)-tagged cDNA was inserted into the lentiviral transfer vector FSW under the control of the neuron-specific synapsin I promoter. Lentiviral vectors were also made expressing short hairpin interfering RNA (shRNA) against murine ASK1 or PRX 1-4 (Table 1). The gene-specific targeting sequence (ASK1t, or PRX1t, PRX2t, PRX3t, and PRX4t) or its counterpart scramble sequence ( $A S K 1 s c$ or $P R X s c$ ) was inserted into the transfer vector FSW under the control of the U6 promoter. The constructed transfer vectors were transformed into Stbl3 Escherichia coli and then isolated using the EndoFree Plasmid Maxi Kit (Qiagen). Large-scale production of the virus was performed using a protocol described previously (Stetler et al.,
2008). In brief, a plasmid mixture containing $435 \mu \mathrm{g}$ of pCMV $\Delta$ R8.9 (packaging construct), $237 \mu \mathrm{g}$ of pVSVG (envelope plasmid), and $675 \mu \mathrm{g}$ of FSW (transfer vector) was suspended in $34.2 \mathrm{ml}$ of $\mathrm{CaCl}_{2}(250 \mathrm{mM})$ and added volume for volume into $2 \times$ BES ( $2 \mathrm{~N}$, N-bishydroxyethyl-2aminoethane-sufonic acid) buffer, $\mathrm{pH}$ 6.95. The $\mathrm{DNA}-\mathrm{CaCl}_{2}$ precipitate was added to human kidney $293 \mathrm{FT}$ cells and allowed to incubate for $12 \mathrm{~h}$ before switching to fresh culture medium. The supernatant was collected $72 \mathrm{~h}$ after transfection, filtered through the $0.45 \mu \mathrm{m}$ filter flask and centrifuged at 21,000 rpm for $2 \mathrm{~h}$. Viruses were further purified by sucrose gradient ultracentrifuge. The pellet was suspended in $3 \mathrm{ml}$ of PBS, loaded on the top of $2 \mathrm{ml}$ of $20 \%$ sucrose solution, and centrifuged at 22,000 rpm for $2 \mathrm{~h}$. The resulting pellet was resuspended in $200 \mu \mathrm{l}$ of DMEM, aliquoted, and stored at $-70^{\circ} \mathrm{C}$. The titer of the vector stock was determined using ELISA.

Gene transfection of MN9D cells. The MN9D cell cultures were infected for $3 \mathrm{~d}$ with Lenti-PRX1, Lenti-PRX2, Lenti-PRX3, Lenti-PRX4, LentiPRX1t, Lenti-PRX2t, Lenti-PRX3t, Lenti-PRX4t, Lenti-PRX2dn, LentiASK1t, Lenti-ASK1 h, Lenti-ASK1dn, or the control vectors [empty vector, Lenti-GFP (green fluorescent protein)]. For each of the shRNAtargeted genes, a scrambled sequence containing the same nucleotide composition in a randomized order was constructed and used as control. Additional control also included an shRNA targeting green fluorescent protein (5'-AACAGCTGCTAGGATTACACA-3'). The overexpression of PRXs and knockdown of PRXs or ASK1 in MD9D cells were confirmed by Western blot analysis.

Cell viability. Cell viability was measured by oxidation of XTT using the Procheck Cell Viability assay kit (Intergen). All values were averaged from at least 12 wells from three independent experiments. Cell viability in some experiments was also measured using Hoechst 33258 staining and terminal deoxynucleotidyl transferase-mediated biotinylated UTP nick end labeling (TUNEL) staining (Weng et al., 2007). The percentages of cells showing chromatin condensation/fragmentation or DNA fragmentation were quantified by counting at least 3000 cells under each experimental condition (three randomly selected fields per well, four to six wells per condition per experiment, and three independent experiments).

Peroxidase activity assay. Peroxidase activity was measured by the consumption of NADPH, which was mediated by E. coli Trx and mammalian Trx reductase at $30^{\circ} \mathrm{C}$ for $10 \mathrm{~min}$ for bacterially expressed proteins and for $1 \mathrm{~h}$ for precipitated proteins from cultured neurons or the SNc. The peroxiredoxin activity assay kit (Redoxica) was used for the measurement according to the manufacturer's instructions. In brief, $250 \mu \mathrm{g}$ of bacterially expressed proteins or the precipitated proteins was incubated with $5 \mathrm{~mm}$ Trx, $1 \mathrm{~mm}$ Trx reductase, and $100 \mathrm{~mm}$ NADPH in HEPES, pH 7.5. The reaction was initiated by the addition of $\mathrm{H}_{2} \mathrm{O}_{2}$ at a final concentration of $0.2 \mathrm{~mm}$. The consumption of NADPH was measured at $340 \mathrm{~nm}$ by spectrophotometer, and the linear rate of decrease in absorbance in the first $120 \mathrm{~s}$ was used for calculation. The data were expressed as percentage changes in PRX activity over control noninjured cell cultures or animals.

ASK1 kinase assays. Cell lysates were prepared under non-denaturing conditions as described previously (Gao et al., 2005), and $150 \mu \mathrm{g}$ of protein was used for each kinase assay. The kinase in cell lysates was captured using the anti-ASK1 antibody (Santa Cruz Biotechnology) and then incubated with recombinant myelin basic protein (MBP) in the presence of $\left[\gamma^{-32} \mathrm{P}\right] \mathrm{ATP}$, and MBP phosphorylation was detected using autoradiogram.

Animal studies. All animal experiments were approved by the University of Pittsburgh Institutional Animal Care and Use Committee and performed in accordance with the National Institutes of Health Guide for the Care and Use of Laboratory Animals. Male C57BL/6 mice (Jackson Laboratory) weighing $\sim 25$ g were placed in a stereotaxic device under $1.5 \%$ isoflurane anesthesia. Animals first received Lenti-PRX2, LentiASK1t, or lentivirus carrying the corresponding scrambled sequences into the left $\mathrm{SN}$ at a rate of $5 \mu \mathrm{l} / 50 \mathrm{~min}$, using the coordinates $2.75 \mathrm{~mm}$ caudal and $0.8 \mathrm{~mm}$ lateral to bregma, at a depth of $3.7 \mathrm{~mm}$ from the dural surface. Three weeks later, $3 \mu \mathrm{g}$ of 6-OHDA (in $1.4 \mu \mathrm{l}$ of saline) or saline alone was injected into the left striatum. Mice were killed at different time points following 6-OHDA for biochemical or histological assessment. 
Table 2. Postmortem human midbrain samples from PD and control subjects

\begin{tabular}{llllll}
\hline & $N$ & Age $(\mathrm{yr})$ & PMI (h) & Male/female ratio & Braak Tau stage \\
\hline Control & 12 & $72.5 \pm 13$ & $5.5 \pm 3.5$ & $3: 1$ & 2.8 \\
PD & 12 & $80.9 \pm 6.4$ & $8.7 \pm 5.7$ & $3: 1$ & 0.0 \\
\hline
\end{tabular}

The overexpression of PRX2 or knockdown of ASK1 in neurons was confirmed by immunocytochemistry and Western blot analysis.

Behavioral testing. Behavioral testing was performed using previously published protocols (Signore et al., 2006; Weng et al., 2007). Apomorphine-induced rotations were monitored 1 week before Lesioning, and at 2 and 3 weeks or at 8 weeks following 6-OHDA lesioning. Mice were given a subcutaneous injection of apomorphine $(0.1 \mathrm{mg} / \mathrm{kg}$ in physiological saline), placed individually in plastic beakers (diameter: $13 \mathrm{~cm}$ ), and videotaped from above for $30 \mathrm{~min}$. Quantitative analyses of completed $\left(360^{\circ}\right)$ left and right rotations were made off-line by an investigator blinded to the experimental group assignment of the animals. The corner test was used to assess spontaneous turning. A trial consisted of noting the animal's preference for spontaneously rearing and turning to the right or left when encountering a $30^{\circ}$ corner composed of two movable opaque Plexiglas walls. Mice were tested 1 week before 6-OHDA and then at 3 or 8 weeks following 6-OHDA. Each testing period included 10 trials.

Measurements of catecholamine levels. The striata of mice were removed at 3 weeks following 6-OHDA administration, immediately frozen on dry ice, and stored at $-80^{\circ} \mathrm{C}$. HPLC with electrochemical detection was used to measure dopamine and its metabolites (Larsen et al., 2002). Catecholamine concentrations are expressed as picomoles per milligrams of fresh striatal tissue.

Western blots. Protein extracts were prepared according to previously published protocols (Cao et al., 2002). Immunoreactivity was semiquantified by gel densitometric scanning and analyzed with the MCID image analysis system (Imaging Research). The primary antibodies used in this study were as follows: mouse anti-tyrosine hydroxylase (TH) (Millipore Bioscience Research Reagents), rabbit antibodies t-ASK1 (total ASK1), p-ASK1 (phosphorylated-ASK1), PRX2, PRX-SO 3, Trx, t-p38 (total p38), p-p38 (phosphorylated-p38), t-JNK (total JNK), p-JNK (phosphorylated-JNK), t-MKK4 (total MKK4), p-MKK4 (phosphorylated MKK4), t-MKK6 (total MKK6), p-MKK6 (phosphorylated MKK6), p-cJun (phosphorylated c-Jun), $\beta$-actin, active caspase-3 and -9 (Cell Signaling Technology), rabbit cytochrome $c$, and HA (Santa Cruz Biotechnology).

Postmortem human samples. Midbrain tissues from 12 control and 12 PD subjects, each with a 3:1 male/female ratio (Table 2), were obtained from the University of Pittsburgh Brain Bank under a protocol approved by the University of Pittsburgh Committee on Research Involving the Dead. The control group consisted of nine normal subjects and three pure Alzheimer disease patients with normal midbrain sections; as there were no significant differences observed, these two subgroups were combined for statistical analysis. Cognitively normal, aged individuals typically exhibit Braak tangle stages $<4$ (Braak and Braak, 1991; Knopman et al., 2003). PD staging was performed by $\alpha$-synuclein immunohistochemistry as described previously (Braak et al., 2003).

Immunohistochemistry. Animals were killed at the indicated time points after 6-OHDA administration. Coronal sections of $30 \mu \mathrm{m}$ thickness were made from brains following the fixation procedures described previously (Signore et al., 2006; Weng et al., 2007). Using an antibody against TH, DA neurons were identified with biotinylated secondary antibody and Vectastain $\mathrm{ABC}$ reagents (Vector Laboratories). After washing, the bound complex was visualized by a diaminobenzidine staining kit (Vector Laboratories). For double-label immunofluorescence staining, sections were first incubated with rabbit anti-p-c-Jun, anti-HA, or anti-ASK1 antibody (Cell Signaling Technology) at $4^{\circ} \mathrm{C}$ for $24 \mathrm{~h}$, followed by incubation for $2 \mathrm{~h}$ at room temperature with goat anti-rabbit Cy3 immunoconjugate (Jackson ImmunoResearch). Sections were then subjected to immunofluorescent staining against TH. For the assessment of nonspecific staining, alternating sections from each experimental condition were incubated without the primary antibodies.
Human tissues were stained for phosphokinases as previously described (Zhu et al., 2002). In brief, deparaffinized sections, treated with $3 \% \mathrm{H}_{2} \mathrm{O}_{2}$ for $10 \mathrm{~min}$, were incubated at $4^{\circ} \mathrm{C}$ overnight with rabbit antip-p38 (1:300, Cell Signaling Technology), anti-p-JNK antibodies (1:100, Cell Signaling Technology) or anti-p-ASK1 (1:100, Cell Signaling Technology), followed by biotinylated anti-rabbit IgG (1:500; Jackson ImmunoResearch) at $25^{\circ} \mathrm{C}$ for $1 \mathrm{~h}$, and either streptavidin-horseradish peroxidase (1:500, Jackson ImmunoResearch) for p-JNK and p-p38 or the Vectastain avidin biotin system PK-6100 kit (Vector Laboratories) for p-ASK1. The peroxidase reaction was visualized with 3-amino-9ethylcarbazole substrate (BioGenex). For double-label immunofluorescence, sections were initially incubated with phosphokinase antibodies (1:100 with heat-citrate/pepsin antigen retrieval), followed by Alexa Fluor-488-conjugated $2^{\circ}$ antibodies. The sections were rinsed with PBST and then incubated with mouse anti- $\alpha$-synuclein (1:1500, BD Biosciences), followed by Cy3-conjugated anti-mouse antibody (Jackson ImmunoResearch). For negative controls, the primary antibodies were replaced with nonimmune rabbit or mouse IgG. The slides were observed using a Molecular Dynamics laser-scanning confocal imaging system. SNc neurons were identified and analyzed as described previously (Zhu et al., 2002). The SNc neurons with or without p-p38, p-JNK, or p-ASK1 immunostaining were expressed as a percentage of total $\mathrm{SNc}$ neurons for each case.

Stereological cell counting. The Bioquant Image Analysis program was used as described previously (Weng et al., 2007) to count SNc DA neurons that were immunopositive for $\mathrm{TH}$. The entire $\mathrm{SNc}$ from a given section was captured with a color charge-coupled device camera, and grid squares of $50 \times 50 \mu \mathrm{m}$ were generated over the region of interest. A $25 \times 25 \mu \mathrm{m}$ counting frame and a focus depth of $25 \mu \mathrm{m}$ in a $40 \mu \mathrm{m}$ section (a dissector) were used. An average of nine dissectors per section yields the desired number of counted neurons throughout the SNc. The rostral-caudal length of the SNc was $4 \mathrm{~mm}$, and every fourth section was counted. The total number of neurons was calculated using the optical dissector, equal to the quotient of the total number of neurons counted and the product of the fractions for sampling section frequency (SSF, fraction of sections counted), area section frequency (ASF, sampling area/area between dissectors), and thickness section frequency (TSF, dissector depth/section thickness), or $n=\sum$ neurons counted $\times 1 /$ SSF $\times$ $1 / \mathrm{ASF} \times 1 / \mathrm{TSF}$. For our study, SSF $=$ one of four sections, $\mathrm{ASF}=25 \times$ $25 \mathrm{~mm} / 50 \times 50 \mathrm{~mm}$, and TSF $=25 \mathrm{~mm} / 40 \mathrm{~mm}$.

Statistical analysis. All data are reported as the mean \pm SEM. Statistical significance between multiple groups was performed using one-way ANOVA. When ANOVA showed a significant difference, post hoc Bonferroni/Dunn tests for multiple comparisons were performed. A value of $p<0.05$ was considered statistically significant.

\section{Results \\ PRX2 protects against 6-OHDA neurotoxicity in DA neurons in vitro}

To determine which PRXs may affect the demise of DA neurons following PD-relevant neurotoxic challenge, we designed a series of lentiviral constructs to either overexpress or knock down the expression of 2-Cys PRXs individually (PRX1, PRX2, PRX3, or PRX4) (supplemental Fig. 1, available at www.jneurosci.org as supplemental material). The constructs were tested for their effects on 6-OHDA-induced cell death in neuronal differentiated MN9D DA cells. As shown (Fig. $1 A, B$ ), 6-OHDA at 50 and 100 $\mu \mathrm{M}$ resulted in $\sim 50$ and $75 \%$ cell death, respectively. Overexpression of PRX1, PRX2, or PRX4 but not PRX3 significantly attenuated 6-OHDA neurotoxicity. In contrast, knockdown of PRX1, 
A

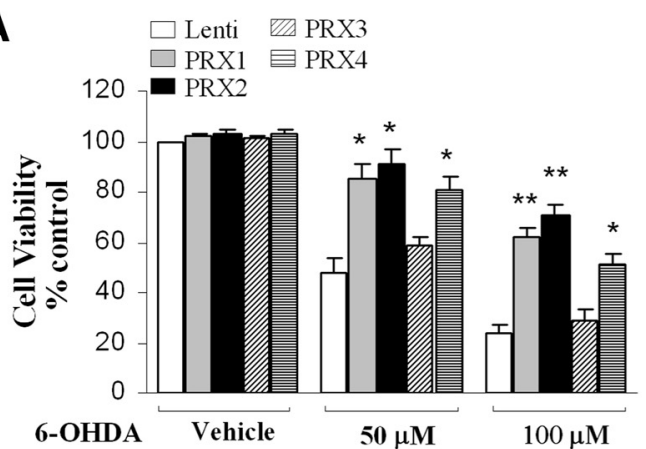

B

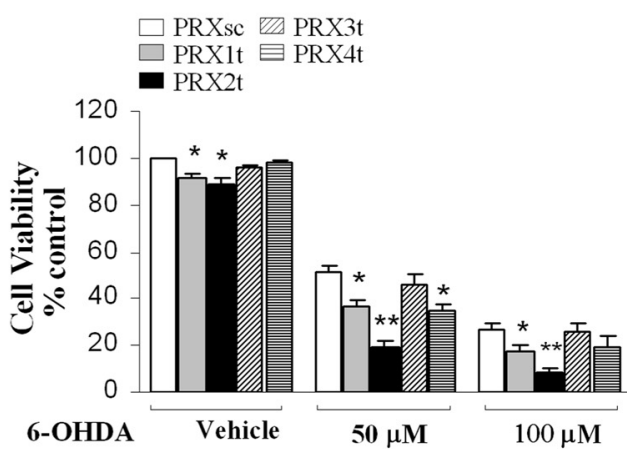

C

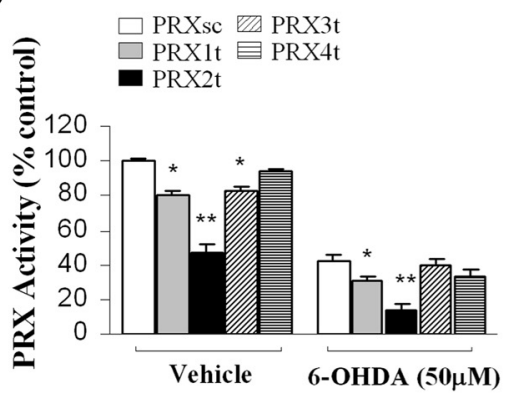

E

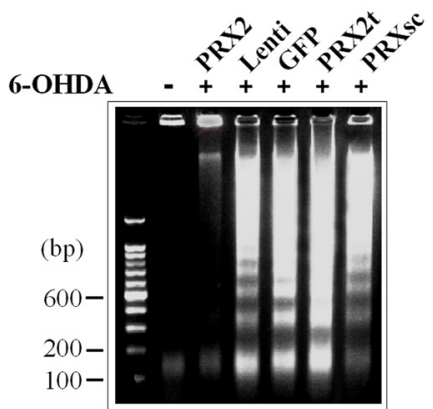

G

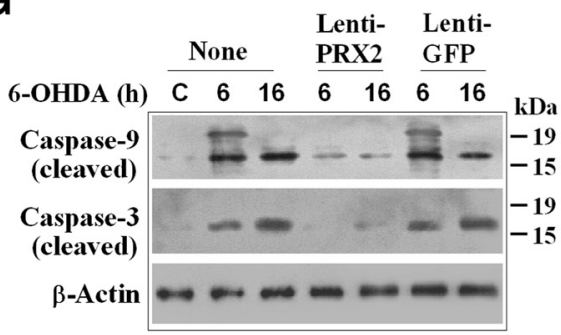

D

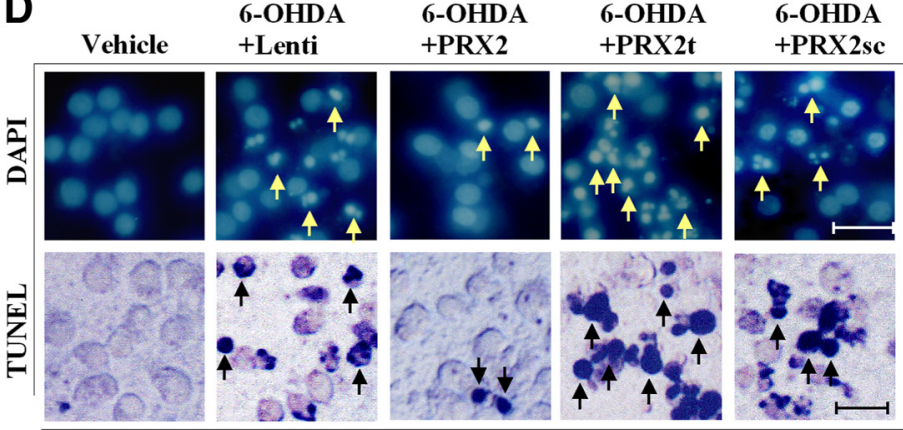

F

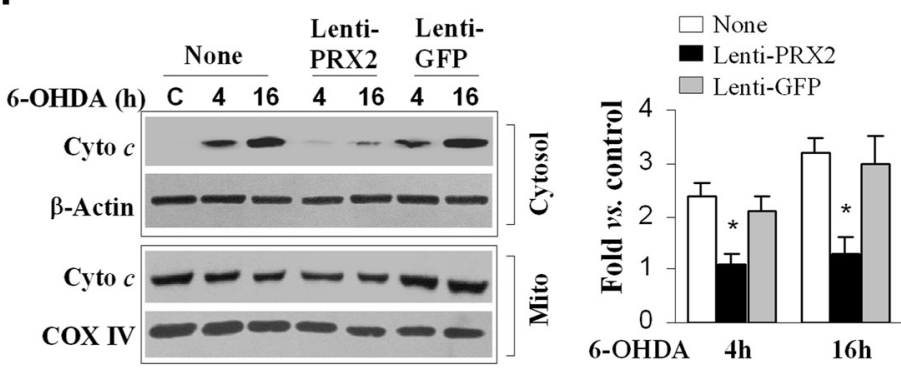

H

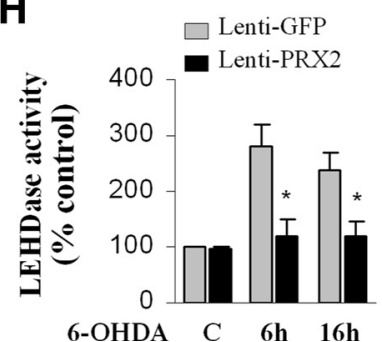

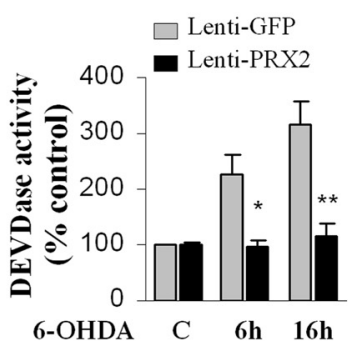

Figure 1. Cellular 2-Cys PRXs protect against 6-OHDA neurotoxicity in MN9D cells. A, Neuronal-differentiated MN9D cells were infected for $3 \mathrm{~d}$ with lentiviral vectors containing cDNA of human PRX1, PRX2, PRX3, PRX4, or the empty vector, and exposed to 6-OHDA at the indicated concentrations. Cell viability was determined at $24 \mathrm{~h}$ after $6-0 \mathrm{HDA}$ exposure. ${ }^{*} p<0.05$, ${ }^{* *} p<0.01$ versus empty vector controls; $n=12$ from 3 independent experiments. $B$, MN9D cells were transfected for $3 \mathrm{~d}$ with lentiviral vectors containing shRNA targeting PRX1 (PRX1t), PRX2 (PRX2t), PRX3 (PRX3t), PRX4 (PRX4t), or the PRX2 scramble control sequence (PRXsc), and exposed to 6-0HDA. Cell viability was determined at $24 \mathrm{~h}$ after 6-0HDA exposure. ${ }^{*} p<0.05,{ }^{* *} p<0.01$ versus PRXsc controls; $n=12$ from three independent experiments. $C$, Under the conditions of $\boldsymbol{B}$, total cellular PRX activity was determined at $2 \mathrm{~h}$ after $6-0 \mathrm{HDA}(50 \mu \mathrm{m})$ or vehicle. ${ }^{*} p<0.05,{ }^{* *} p<0.01$ versus PRXsc controls; data from four experiments. D, Nuclear staining (DAPI) and TUNEL staining at $24 \mathrm{~h}$ after 6-OHDA (50 $\mu \mathrm{m}$ ) in MN9D cells infected with lentiviral vectors for PRX2 overexpression (PRX2) or knockdown (PRX2t). Note that PRX2 overexpression attenuates, whereas PRX2 knockdown promotes, nuclear apoptosis and DNA fragmentation (arrows) following 6-0HDA exposure. E, PRX2 overexpression attenuates, whereas PRX2 knockdown promotes, apoptotic DNA fragmentation $24 \mathrm{~h}$ after 6-0HDA exposure, as determined using DNA gel electrophoresis. The gel is representative of three experiments with similar results. $\boldsymbol{F}$, PRX2 overexpression attenuates cytochrome c release at 4 and $16 \mathrm{~h}$ after 6-0HDA (50 $\mu \mathrm{m}$ ) exposure, determined by subcellular fractionation and immunoblotting. Right, The graph illustrates the cytosolic cytochrome c after 6-OHDA in cells infected with Lenti-PRX2 or the control vector Lenti-GFP. ${ }^{*} p<0.05$ versus noninfected controls; $n=$ 4/condition. $\mathbf{G}, \boldsymbol{H}$, PRX2 overexpression attenuates the activation of caspase-9 and caspase- 3 at 6 and $16 \mathrm{~h}$ after 6-0HDA (50 $\mu \mathrm{m})$ exposure, determined by immunoblotting against cleaved caspases (G) and peptide substrate-based protease activity assays (LEHD for caspase-9, DEVD for caspase-3) $(\boldsymbol{H})$, respectively. ${ }^{*} p<0.05 ;{ }^{* *} p<0.01$ versus Lenti-GFP-infected controls, $n=4 /$ condition. Cyto, Cytochrome; COX, cyclo-oxygenase.

PRX2, or PRX4 by targeting with the appropriate shRNA showed significant enhancement in neurotoxicity of 6-OHDA. The knockdown of PRX2 had the greatest deleterious effect on cell viability and significantly potentiated the neurotoxicity induced by either 50 or
$100 \mu \mathrm{M}$ 6-OHDA. In contrast, knockdown of PRX3 expression failed to significantly affect 6-OHDA-induced cell death.

When total PRX activity was measured in neuronal differentiated MN9D cells before and following $50 \mu \mathrm{M}$ 6-OHDA (Fig. 
A

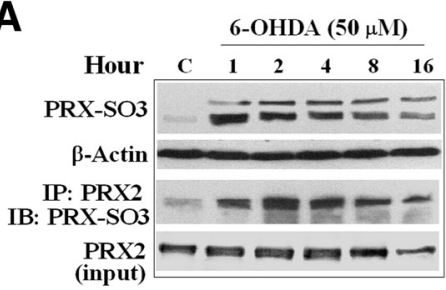

B

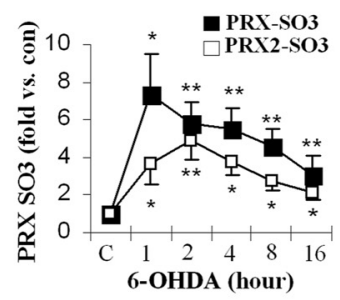

C

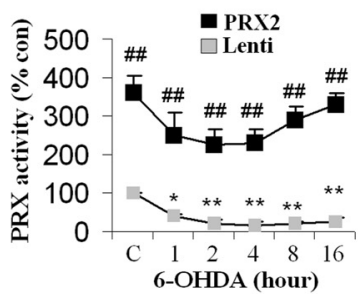

D

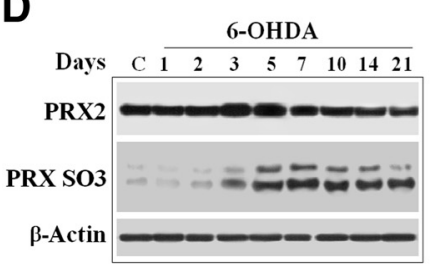

E

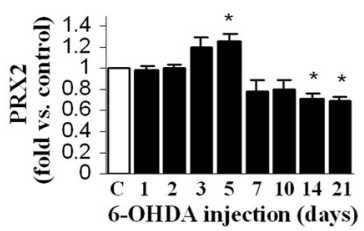

$\mathbf{F}$

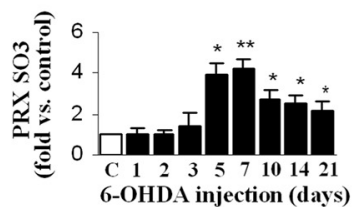

G

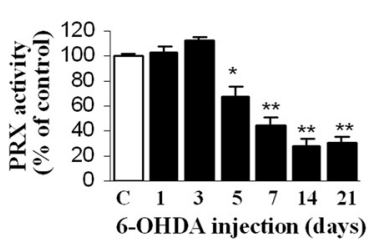

H Lenti Lenti-PRX2 L

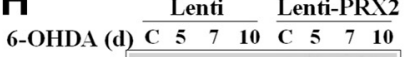

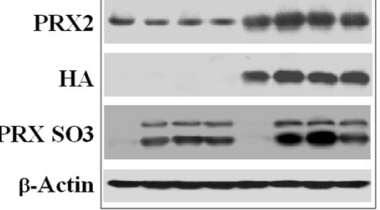

I

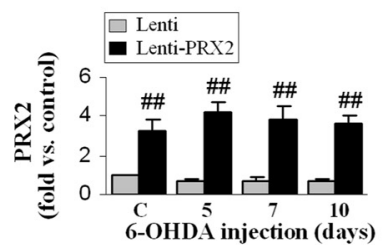

J

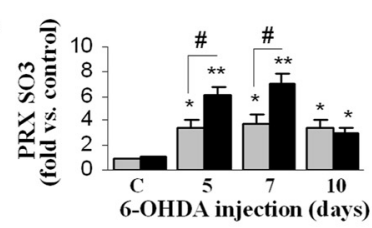

K

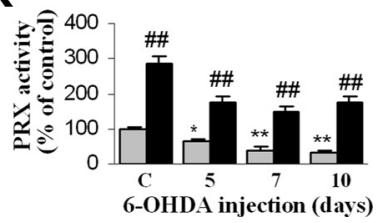

HA-PRX2
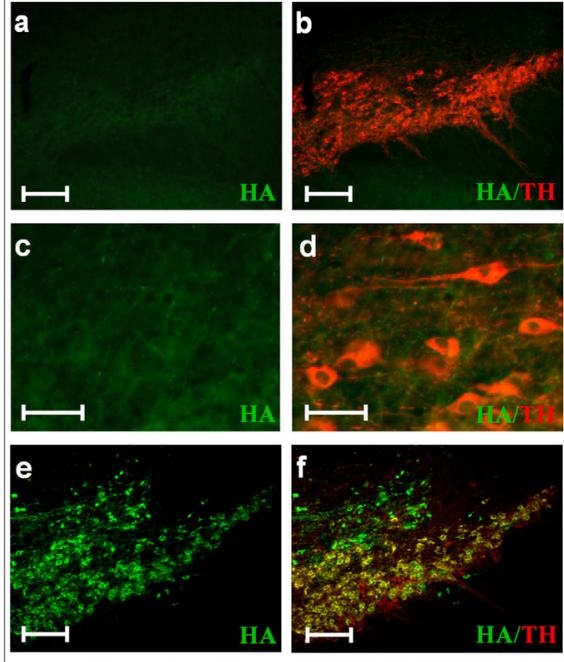

g
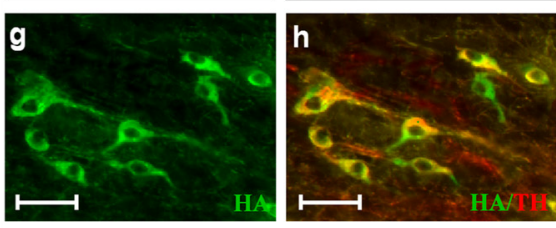

Figure 2. Lentiviral overexpression of PRX2 enhances PRX activity and PRX oxidation in DA neurons. $\boldsymbol{A}, \boldsymbol{B}, 6-0 \mathrm{OHDA}$ induces PRX oxidation in MN9D cells. Neuronal-differentiated MN9D cells were treated with 6-OHDA $(50 \mu \mathrm{m})$. At the indicated time points, cell extracts were subjected to immunoblotting against PRX-SO $\mathrm{S}_{3}$ or PRX2 immunoprecipitation followed by immunoblotting against

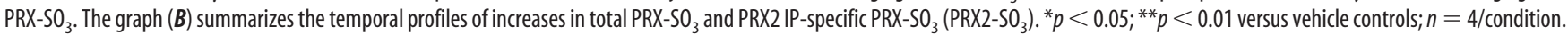
C, MN9D cells were infected with Lenti-PRX2, the empty vector (Lenti) or Lenti-GFP (data not shown) for $3 \mathrm{~d}$, and then challenged with 6-0HDA. Cellular PRX activity was measured at the indicated time points, and data were expressed as percentage changes over vehicle controls. ${ }^{*} p<0.05,{ }^{* *} p<0.01$ versus vehicle controls; \#\# $<0.01$ versus Lenti vector-infected cells; $n=3 /$ condition. D-G, 6-OHDA lesioning induces PRX oxidation and reduced PRX activity in mouse SNc. Mice were subjected to striatal infusion of 6-0HDA (3.0 $\mu \mathrm{g}$ ), and at the indicated time points the SNc extracts were processed for immunoblotting against PRX2 $(\boldsymbol{D}, \boldsymbol{E}), \mathrm{PRX}-\mathrm{SO}_{3}(\boldsymbol{D}, \boldsymbol{F})$, and the PRX activity assay $(\boldsymbol{G}) .{ }^{*} p<0.05,{ }^{* *} p<0.01$ versus saline-injection controls; $n=5-6 /$ group. $\boldsymbol{H}-\boldsymbol{K}$, Lenti-PRX2 gene delivery increases PRX activity and PRX oxidation in mouse SNc. Mouse SNc was infected for $21 \mathrm{~d}$ with empty lentiviral vector (Lenti) or Lenti-PRX2 (HA-tagged), and then received striatal infusion of 6-0HDA $(3.0 \mu \mathrm{g})$ or saline of the same volume. SNc extracts were processed for immunoblotting against PRX2 $(\boldsymbol{H}, \boldsymbol{I}), \mathrm{HA}(\boldsymbol{H}), \mathrm{PRX}-\mathrm{SO} \mathbf{}_{3}(\boldsymbol{H}, \boldsymbol{J})$, and the PRX activity assay $(\boldsymbol{K}) .{ }^{*} p<0.05$, ${ }^{* *} p<0.01$ versus saline controls; $\# p<0.05$; \#\# $<0.01$ versus empty lentivirus group; $n=5-6 /$ group. $L$, Double-label immunofluoroscent staining for HA (HA-tagged PRX2, green) and TH (red) in the SNc at $21 \mathrm{~d}$ after brain inoculation with either empty lentiviral vector ( $\boldsymbol{a}, \boldsymbol{b}$, low magnification; $\boldsymbol{c}, \boldsymbol{d}$, high magnification) or Lenti-PRX2 (e, $\boldsymbol{f}$, low magnification; $\boldsymbol{g}, \boldsymbol{h}$, high magnification). Scale bars: $\boldsymbol{a}, \boldsymbol{b}, \boldsymbol{e}, \boldsymbol{f}, 100 \mu \mathrm{m} ; \boldsymbol{c}, \boldsymbol{d}, \boldsymbol{g}, \boldsymbol{h}, 30 \mu \mathrm{m}$.

1C), a 50-70\% reduction in PRX activity in toxin-exposed cells was observed. The 6-OHDA-induced decrease of PRX activity was enhanced by knockdown of PRX1 or PRX2, consistent with the beneficial effect of PRX1 or PRX2 on cell viability. Dictated by its relatively low abundance in MN9D cells (supplemental Fig. $1 D, H$, available at www.jneurosci.org as supplemental material), knockdown of PRX4 did not affect total cellular PRX activity. PRX3 knockdown, which decreased total PRX activity under control conditions, did not further decrease PRX activity under neurotoxic stress. Together, these results suggest that PRX2, PRX1, and PRX4 all aid DA neurons in surviving the 6-OHDA neurotoxic challenge; however, PRX2 appears to afford the greatest endogenous neuroprotection. Because of these results and previously published evidence that $\mathrm{PRX} 2$ is the most abundant PRX enzyme in brain neurons with implications in PD pathogenesis (Fang et al., 2007; Qu et al., 2007), we focused the present study on investigating the role of PRX2 in the response of DA neurons to neurotoxic stress.

Previous studies using neuronal-differentiated MN9D cells have shown that 6-OHDA induces caspase-dependent cell death via the intrinsic apoptotic pathway (Signore et al., 2006; Weng et al., 2007). Thus, we examined the ability of PRX2 to inhibit neuronal apoptosis and the apoptotic traits activated specifically for the intrinsic pathway. As illustrated (Fig. 1D,E; supplemental Fig. 2A-D, available at www.jneurosci.org as supplemental material), PRX2 protected against MN9D cell death, determined by 
assessing apoptotic DNA fragmentation at $24 \mathrm{~h}$ after 6-OHDA. Consistent with our previous reports, 6-OHDA induced mitochondrial release of cytochrome $c$ in MN9D cells preceding cell death. Cultures overexpressing PRX2 showed significant attenuation of cytochrome $c$ release (Fig. $1 F)$. Exposure of MN9D cells to 6-OHDA also led to the activation of both caspase- 9 and caspase-3. Again, PRX2 overexpression nearly completely inhibited the activation of caspase- 9 and caspase- 3 at 6 and $16 \mathrm{~h}$ following neurotoxin exposure (Fig. $1 G, H)$. Thus, PRX2 inhibits 6-OHDAinduced apoptosis of neuronal-differentiated MN9D cells by preventing the activation of the intrinsic apoptotic pathway in DA neurons.

\section{PRX2 overexpression reverses} 6-OHDA-induced PRX inactivation and enhances PRX overoxidation in DA neurons in vitro and in vivo

When acting as a scavenger during oxidative stress, PRXs are readily oxidized at a peroxidatic cysteine residue to generate a sulfenic acid with a consequent reduction in antioxidative activity. The sulfenic acid then reacts with another active PRX2 to form a disulfide bond; or, under heavy oxidative stress, it is overoxidized to form cysteine sulfinic acid (Cys- $\mathrm{SO}_{2}$ ) or cysteine sulfonic acid (Cys- $\mathrm{SO}_{3}$ ) (Low et al., 2008). A recent study suggested that the oxidative status of PRXs dramatically influences their cytoprotective capacity (Lee et al., 2008). We examined the oxidation of PRXs following 6-OHDA challenge using an antibody that specifically recognizes overoxidized PRX (PRX-SO $\mathrm{S}_{3}$ ). Total $\mathrm{PRX}-\mathrm{SO}_{3}$ was barely detectable in MN9D cells without 6-OHDA, but was remarkably enhanced as early as $1 \mathrm{~h}$ following 50 $\mu \mathrm{M}$ 6-OHDA treatment and remained at increased levels for at least $16 \mathrm{~h}$ (Fig. $2 A, B)$. To further determine the oxidative modification of PRX2, whole-cell lysates from MN9D cells with or without 6-OHDA treatment were subjected to immunoprecipitation (IP) using the PRX2 antibody followed by immunoblot with anti-PRX-SO . The elevation of PRX2-associated PRX-SO $\left(\mathrm{PRX} 2-\mathrm{SO}_{3}\right)$ occurred from 1 to $16 \mathrm{~h}$ after 6-OHDA, coinciding with the increases in total $\mathrm{PRX}-\mathrm{SO}_{3}$. Indeed, the increases in $\mathrm{PRX} 2-\mathrm{SO}_{3}$ contributed a larger portion of total $\mathrm{PRX}-\mathrm{SO}_{3}$ in MN9D cells following 6-OHDA treatment (Fig. $2 \mathrm{~B}$ ); this is consistent with the observation that PRX2 is the predominant PRX in MN9D cells in response to oxidative stress. The activity analysis demonstrated a decrease in PRX activity that paralleled the increase of $\mathrm{PRX}-\mathrm{SO}_{3}$ following 6-OHDA. Overexpression of PRX2 reversed 6-OHDA-induced PRX inactivation occurring from 1 to $16 \mathrm{~h}$ (Fig. 2C).
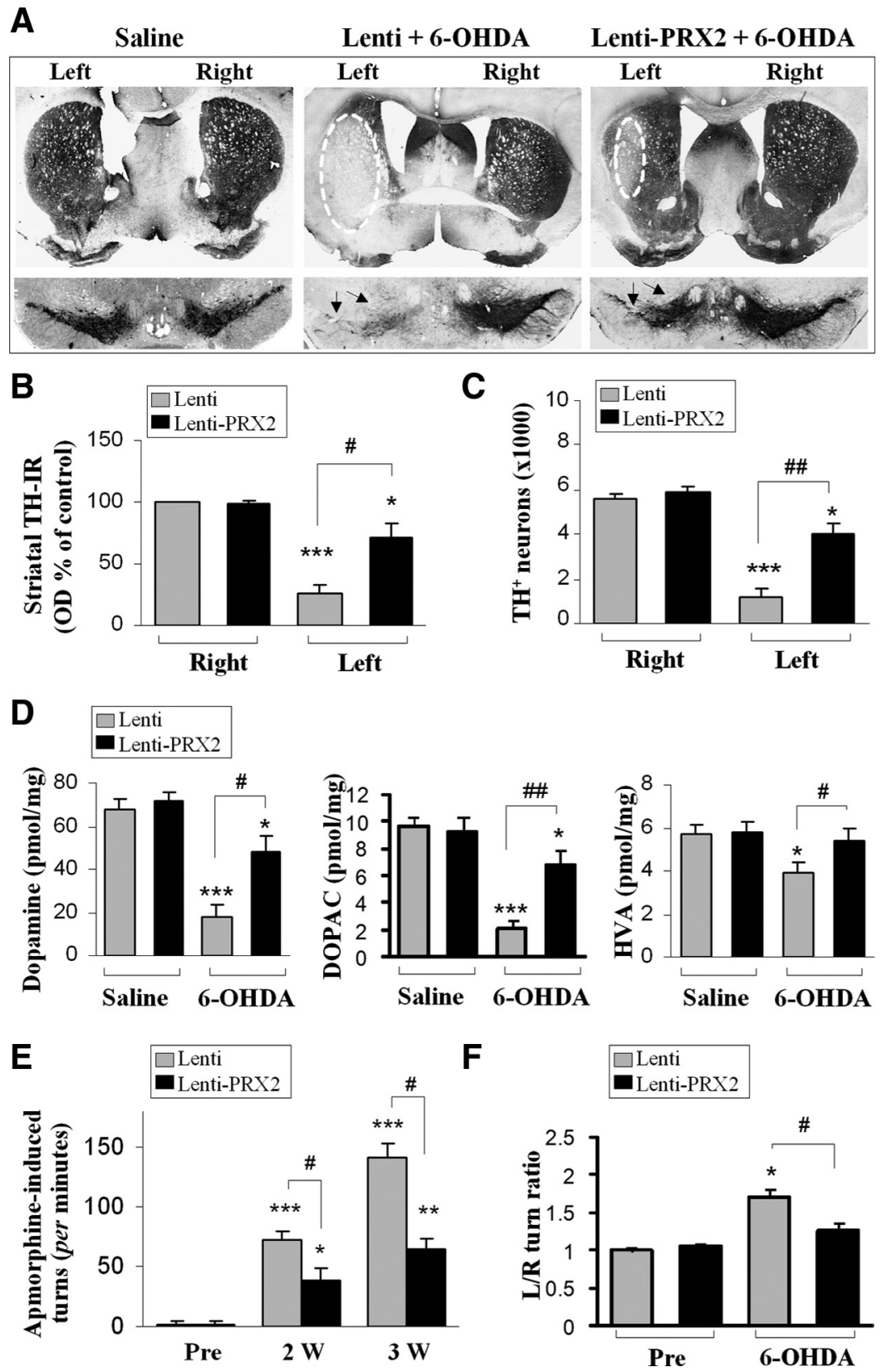

Figure 3. $\mathrm{PRX2}$ protects the nigrostriatal dopaminergic system from 6-OHDA-induced degeneration in mice. $\boldsymbol{A}$, Brain sections from the striatum and SNc showing TH immunoreactivity 3 weeks after striatal infusion of saline or 6-0HDA (3.0 $\mu \mathrm{g}$ ) preceded by brain inoculation of either empty lentiviral vector (Lenti) or Lenti-PRX2. Arrows indicate the SNc region lesioned by 6-0HDA. $\boldsymbol{B}$, Optical measurement of striatal TH immunoreactivity 3 weeks after the injections indicated. Controls were injected with saline. $\boldsymbol{C}$, The numbers of TH-positive neurons in the ipsilateral SNc (injected side) were counted using nonbiased stereological methods 3 weeks after the injections indicated. D, HPLC-electrochemical detection data of catecholamine levels from the ipsilateral striatum 3 weeks following the indicated injections. Catecholamines are expressed as picomoles per milligram fresh, wet tissue. $\boldsymbol{E}$, Apomorphine-induced circling before 6-0HDA injection (Pre) or 2 and 3 weeks after the injections indicated is shown as the number of turns in the first $30 \mathrm{~min}$ after intraperitoneal injection of apomorphine. $\boldsymbol{F}$, Spontaneous turning using the corner test before 6-OHDA injection (Pre) or 3 weeks after the injections indicated. The mean of left/right turns ratio of 10 trials/session are reported. ${ }^{*} p<0.05,{ }^{* *} p<0.01,{ }^{* * *} p<0.001$ versus contralateral or saline control; $\# p<0.05$, \#\#p $<0.01$ versus 6-0HDA plus empty vector (Lenti). $n=10-12$ per group for all experiments.

We confirmed the effect of 6-OHDA on the activity and oxidation of PRXs in vivo. In agreement with our in vitro observations, the levels of PRX-SO 3 in $\mathrm{SNc}$ extracts were markedly increased from 5 to $21 \mathrm{~d}$ after striatal 6-OHDA lesioning (Fig. $2 D, F)$, indicating that PRXs were used as reducing enzymes against oxidative stress in SNc induced by 6-OHDA. The eleva- 
A

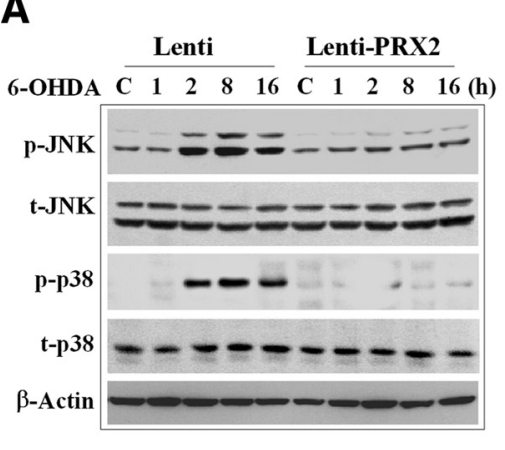

C

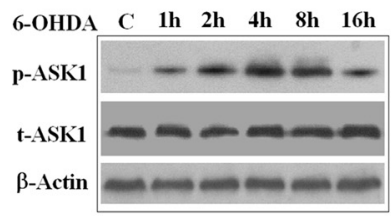

D

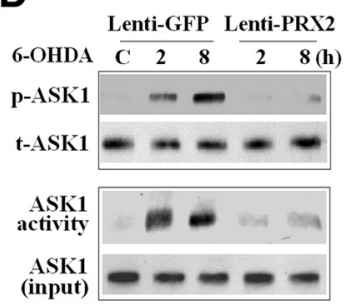

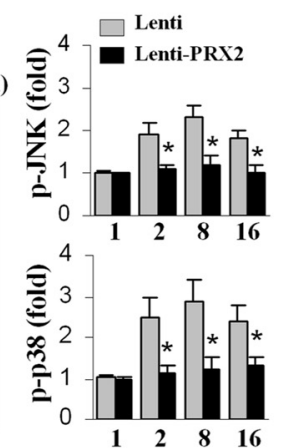

B

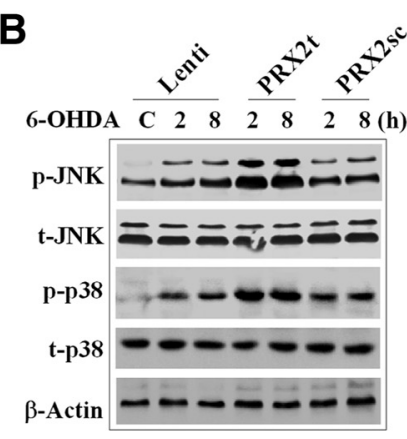

$\mathbf{E}$
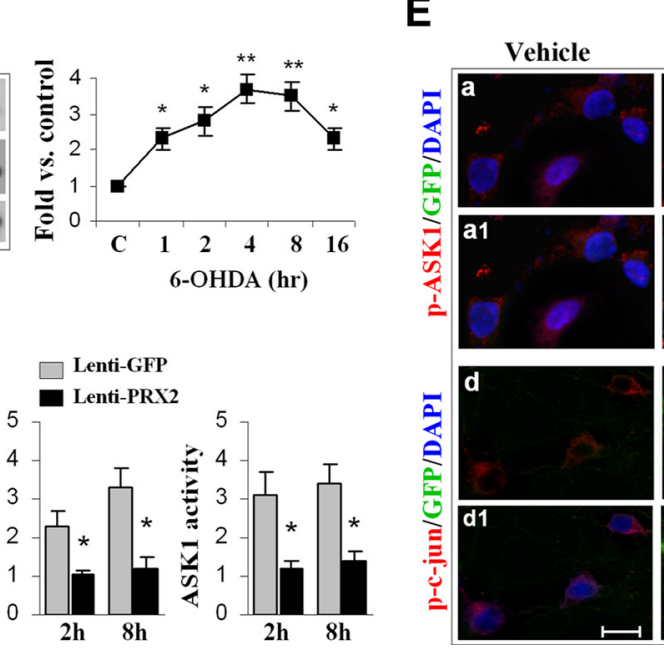
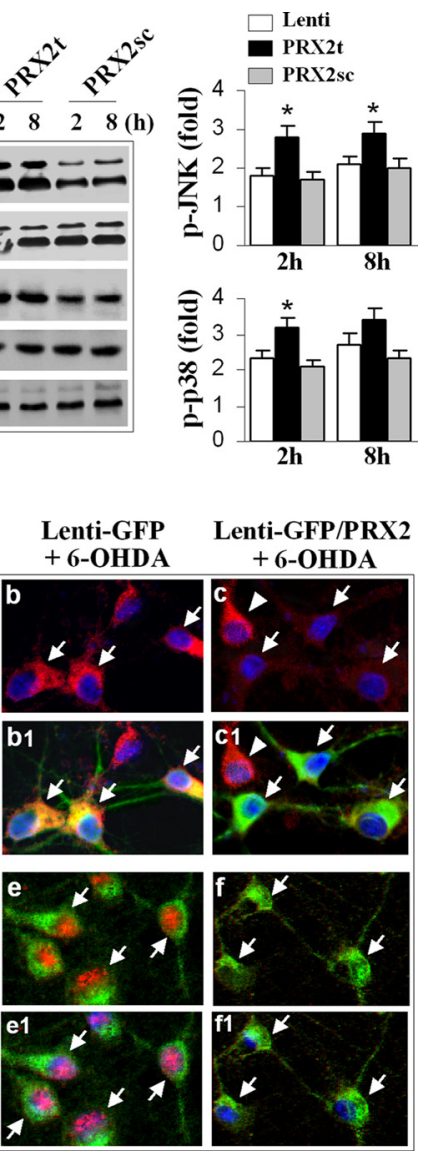

Figure 4. PRX2 inhibits 6-OHDA-induced activation of ASK1 and JNK/p38 signaling pathways in MN9D cells. $A$, Neuronal-differentiated MN9D cells were infected with Lenti-PRX2, the empty vector (Lenti), or Lenti-GFP (data not shown) for $3 \mathrm{~d}$, and then challenged with 6-OHDA $(50 \mu \mathrm{m})$. At the indicated time points, cell extracts were subjected to immunoblotting against p-JNK, total JNK (t-JNK), p-p38, or total $\mathrm{p} 38$ ( $\mathrm{t}-\mathrm{p} 38)$. The fold changes of $\mathrm{p}-\mathrm{JNK}$ and $\mathrm{p}-\mathrm{p} 38$ over the vehicle controls are illustrated in the graphs. ${ }^{*} p<0.05$ versus empty vector control (Lenti); data are from three experiments. B, MN9D cells were infected for $3 \mathrm{~d}$ with lentiviral vectors containing shRNA PRX2t or PRX2sc, or the empty vector (Lenti), and immunoblotting was performed at 2 and $8 \mathrm{~h}$ after 6-0HDA $(50 \mu \mathrm{M})$. The fold changes of $\mathrm{p}$-JNK and p-p38 over the vehicle controls are illustrated in the graphs. ${ }^{*} p<0.05$ versus empty vector control (Lenti) or PRX2sc-infected cells; data are from three experiments. C, 6-OHDA induces ASK1 phosphorylation in MN9D cells in a time-dependent manner. The graph illustrates the temporal profile of p-ASK1 increases after 6-0HDA (50 $\mu \mathrm{M}$ ) exposure. ${ }^{*} p<0.05,{ }^{* *} p<0.01$ versus vehicle control; data are from three experiments. $\boldsymbol{D}$, Under the experimental conditions described in $\boldsymbol{A}$, cell extracts were processed for immunoblotting against $\mathrm{p}$-ASK1 or ASK1 kinase activity assay. The fold changes of p-ASK1 and ASK1 activity over vehicle control are illustrated in the graphs. ${ }^{*} p<0.05$ versus Lenti-GFP; data are from three experiments. E, MN9D cells were infected for $3 \mathrm{~d}$ with Lenti-GFP or both Lenti-GFP and Lenti-PRX2, and then challenged with 6-OHDA (50 $\mu \mathrm{m}$ ). Representative triple-label immunofluorescent images show increased cytosolic p-ASK1 (b, red; $\boldsymbol{b} 1$, yellow, merged with GFP) and nuclear p-c-Jun (e, red; $\boldsymbol{e} 1$, pink, merged with DAPI) immunofluorescence after 6-0HDA; infection with Lenti-PRX2 abolishes the p-ASK1 (cand $\boldsymbol{c} \boldsymbol{1}$ ) and $\mathrm{p}-\mathrm{c}-\mathrm{Jun}(\boldsymbol{f}$ and $\boldsymbol{f} \mathbf{1})$ immunofluorescence after 6-0HDA.

tion of PRX-SO 3 after 6-OHDA lesioning was accompanied by a significant reduction in total PRX activity, beginning at $5 \mathrm{~d}$ and reaching as low as $\sim 30 \%$ of control levels at 14 and $21 \mathrm{~d}$ (Fig. $2 G$ ). In contrast, Western blot analysis revealed no decrease in the expression of PRX2 (Fig. 2E) or PRX1 and PRX4 (data not shown) until 14-21 d after 6-OHDA lesioning, suggesting that the early reduction of total PRX activity in SNc was due to oxidation/disability of PRXs instead of reduced expression levels of the enzymes.

To determine the effect of PRX2 overexpression on PRX activity in vivo, we infused lentivirus vector carrying the human PRX2 (Lenti-PRX2) cDNA or empty lentivirus vector (Lenti) under the control of the neuronal-specific synapsin I promoter into the SNc of mice 3 weeks before 6-OHDA lesioning. SNc protein extracts prepared at 5, 7, and $10 \mathrm{~d}$ after 6-OHDA lesioning were subjected to Western blot analysis, which showed that Lenti-PRX2 successfully increased PRX2 expression and total PRX activity in SNc (Fig. $2 H, I, K$ ). Double-immunofluorescent staining confirmed the expression of HA-tagged PRX2 in THpositive DA neurons of the SNc (Fig. $2 L$ ). Interestingly, Lenti$\mathrm{PRX} 2$ also resulted in a significant increase in $\mathrm{PRX}-\mathrm{SO}_{3}$ levels at 5-7 d after 6-OHDA lesioning (Fig. 2J), suggesting that lentivirus-mediated overexpression of PRX2 added to the antioxidant capacity and activity of SNc against 6-OHDA neurotoxicity.

Together, our in vitro and in vivo data show that 6-OHDA increased overoxidized PRXs and the subsequent loss of PRX activity. The lentiviral overexpression of PRX2 increased both PRX activity and overoxidation levels of PRXs following in vivo 6-OHDA lesioning.

\section{PRX2 overexpression protects DA neurons from 6-OHDA} neurotoxicity in vivo

To substantiate the in vitro findings regarding the neuroprotective effects of PRX2, we injected Lenti-PRX2, Lenti-GFP (data not shown), or empty Lenti vector into the left SNc of mice, followed by an injection of 6-OHDA into the ipsilateral striatum 3 weeks later. Brains were collected 3 weeks after 6-OHDA lesioning. Immunohistochemical staining demonstrated that the 6-OHDA-induced loss of TH-positive neurons in the SNc and the loss of TH-positive neural processes in striatum were remarkably attenuated by PRX2 overexpression (Fig. 3A). Densitometric analysis of TH staining demonstrated that PRX2-overexpressed 


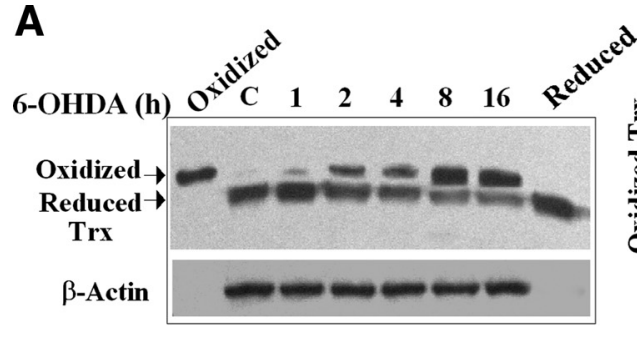

B

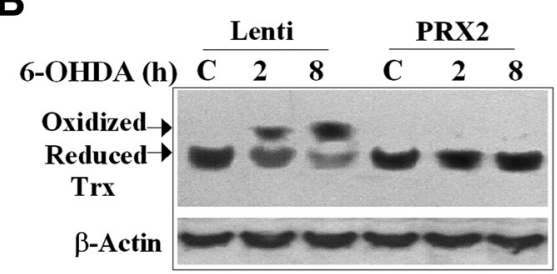

C

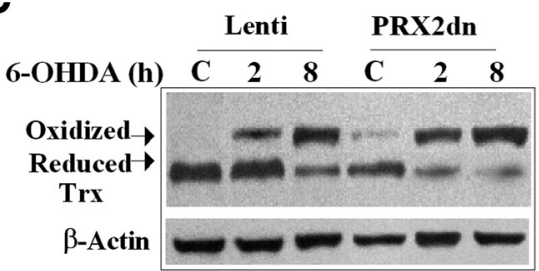

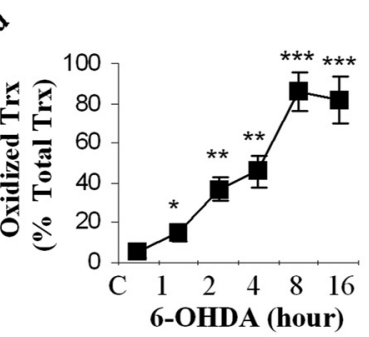

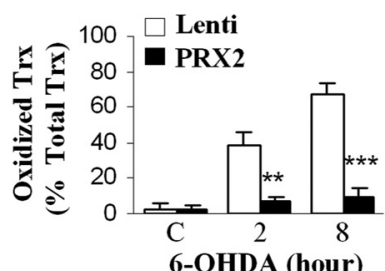

6-OHDA (hour)

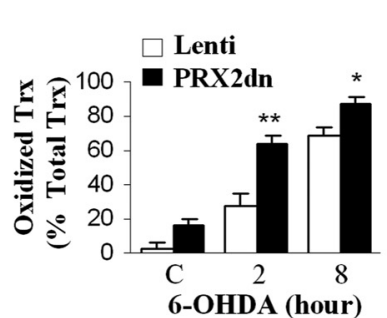

D

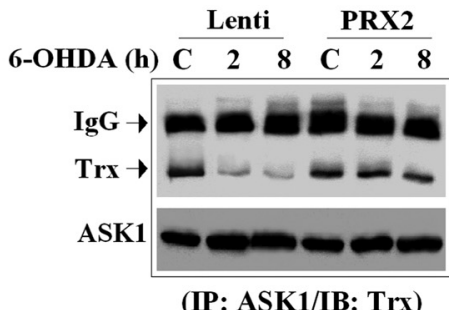

(IP: ASK1/IB: Trx)

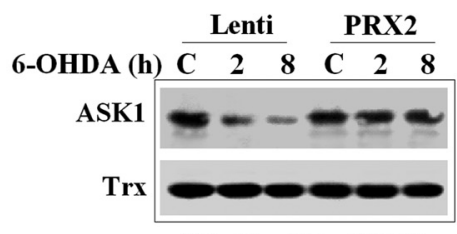

(IP: Trx/IB: ASK1)

E

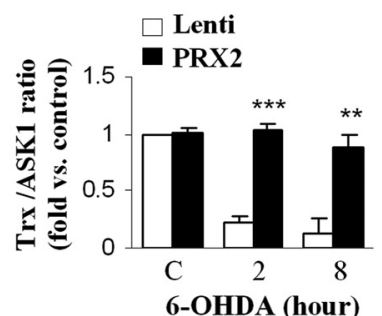

Figure 5. PRX2 inhibits ASK1 activation by regulating the redox state of thioredoxin. $A, 6-0 \mathrm{HDA}$ induces the oxidation of Trx in MN9D cells. Western blot analysis was performed using an antibody recognizing both oxidized and reduced forms of Trx; recombinant oxidized and reduced Trx proteins served as positive controls. The graph illustrates the temporal profile of 6-OHDA (50 $\mu \mathrm{M})$-induced increases of oxidized Trx (percentage of oxidized + reduced Trx). ${ }^{*} p<0.05,{ }^{* *} p<0.01$, ${ }^{* * *} p<0.001$ versus vehicle control; data are from three experiments. B, C, PRX2 inhibits 6-0HDA-induced oxidation of Trx. MN9D cells were infected for $3 \mathrm{~d}$ with an empty vector (Lenti) or Lenti-PRX2 (B), or Lenti-PRX2dn ( $($ ), and immunoblotting against Trx was performed at 2 and $8 \mathrm{~h}$ after $6-0 \mathrm{HDA}$ exposure. Quantitative data on relative levels of oxidized Trx are illustrated in the graphs. ${ }^{*} p<0.05,{ }^{* *} p<0.01,{ }^{* * *} p<0.001$ versus empty vector control; data are from 3 three experiments. $\boldsymbol{D}$, PRX2 prevents the dissociation between Trx and ASK1 following 6-OHDA neurotoxicity. MN9D cells were infected for $3 \mathrm{~d}$ with an empty vector (Lenti) or Lenti-PRX2, and then challenged with 6-OHDA $(50 \mu \mathrm{M})$. Coimmunoprecipitation was performed using antibodies against ASK1 and Trx, respectively. $\boldsymbol{E}$, Quantitative analysis of $\boldsymbol{D}$, top; data are expressed as the relative amounts of Trx that bound to ASK1. ${ }^{* *} p<0.01,{ }^{* * *} p<0.001$ versus empty vector control; data are from three experiments.

animals had more TH-positive DA fibers in the striatum compared with animals treated with the control vector (Fig. $3 B$ ). Stereological quantification of the number of $\mathrm{SNc} \mathrm{TH}$-positive neurons showed that PRX2-overexpressed animals had a greater number of immunopositive neurons after 6-OHDA lesioning compared with control vector-treated animals (Fig. 3C). The neuroprotective effect of PRX2 overexpression against 6-OHDAinduced nigra-striatal degeneration also led to significant elevation, but not complete recovery, of striatal levels of DA and its metabolites [DOPAC (dihydroxyphenylacetic acid) and HVA (homovanillic acid)], determined at 3 weeks after 6-OHDA injection (Fig. 3D).

To determine the impact of PRX2 overexpression on motor functional deficits due to 6-OHDA lesioning, apomorphineinduced ambulatory rotations were tested at 2 and 3 weeks and the corner test for evaluation of spontaneous rearing was performed at 3 weeks after 6-OHDA lesioning. Apomorphine-induced asymmetrical rotations to the contralateral side of 6-OHDA injection were significantly decreased by PRX2 overexpression compared with control vector-treated animals (Fig. 3E). Mice lesioned with 6-OHDA developed a preference for spontaneous turning toward the side contralateral to 6-OHDA injection. Turning was also significantly attenuated in animals overexpressing PRX2 (Fig. 3F).

To determine whether PRX2 overexpression in SNc offers prolonged neuroprotection against 6-OHDA lesioning, in another set of experiments, outcome measurements were performed 8 weeks after $6-O H D A$ lesioning. Compared with control vector-infected mice, Lenti-PRX2-infected mice showed significantly decreased apomorphine-induced asymmetrical rotations or spontaneous turning, and significantly increased TH-positive neuron survival in the $\mathrm{SNc}$ (supplemental Fig. $3 A-C$, available at www.jneurosci.org as supplemental material).

PRX2 inhibits the activation of ASK1 and downstream JNK/p38 signaling pathways in neuronal-differentiated MN9D cells

In the MN9D cell model, we found that although PRX2 reduced 6-OHDA-induced apoptotic cell death, it failed to protect against staurosporine-induced apoptosis (supplemental Fig. 2E-G, available at www.jneurosci.org as supplemental material), suggesting that PRX2 may target specific upstream cell death signaling pathways instead of blocking downstream common apoptosis execution pathways. JNK and p38 MAPK are two well characterized oxidative stress-responsive pro-death signaling pathways that may participate in DA neuronal cell death in PD models (Choi et al., 2004; Klintworth et al., 2007). We then sought to determine whether suppression of either JNK or the p38 MAPK pro-death pathway occurs during PRX2-induced neuroprotection. We confirmed that the prerequisite phosphorylation step for activation of both JNK and p38 increased robustly in MN9D cells 2-16 h after 6-OHDA treatment. Overexpression of PRX2 in MN9D cells inhibited the phosphorylation of JNK and p38 (Fig. 4A), while knockdown of PRX2 enhanced the phosphorylation of both molecules (Fig. 4B). PRX2 overexpression also inhibited 
A
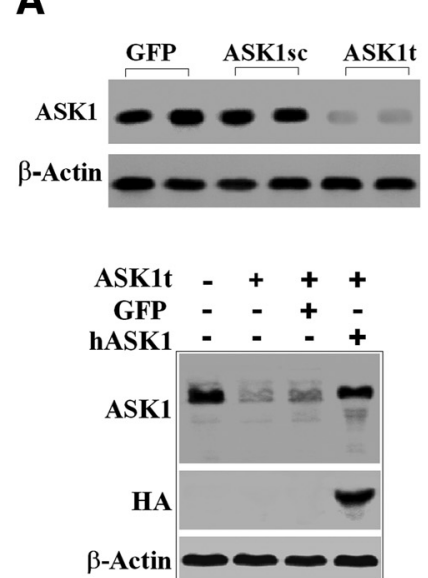

D

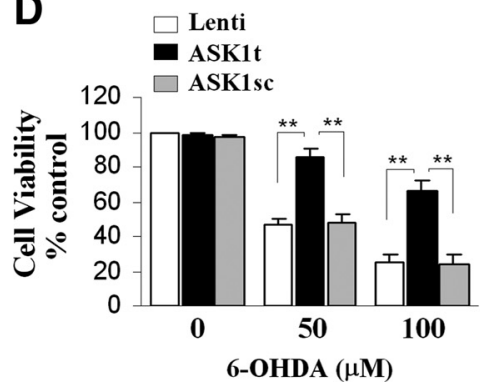

B

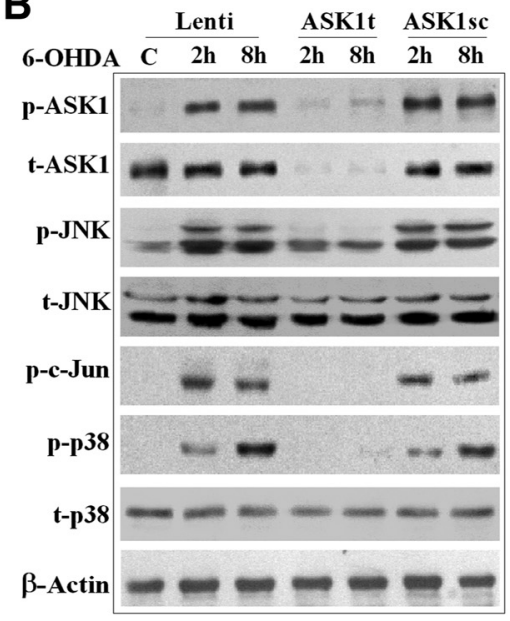

E

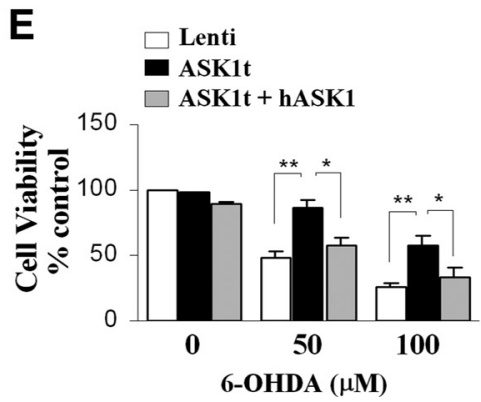

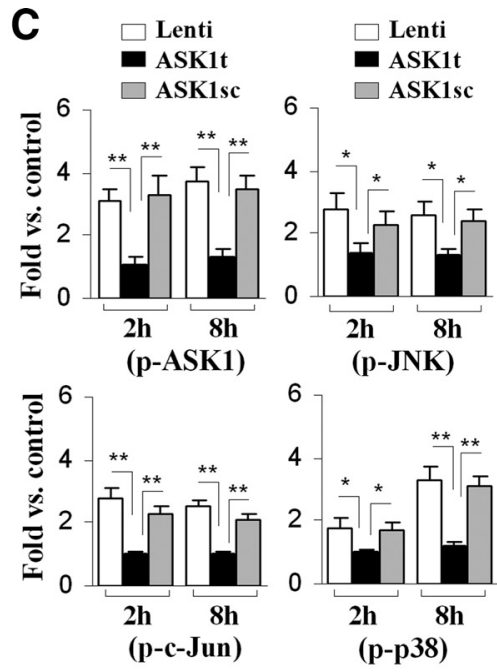

$\mathbf{F}$

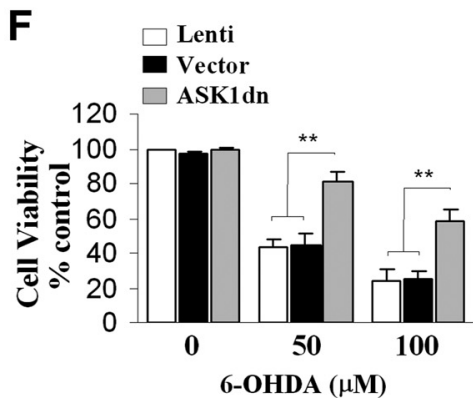

Figure 6. Critical role of ASK1-JNK/p38 signaling pathway in 6-OHDA neurotoxicity in MN9D cells. $A$, Knockdown of ASK1 expression in MN9D cells. MN9D cells were infected for $3 \mathrm{~d}$ with lentiviral vectors containing shRNA ASK1t or ASK1sc, or GFP CDNA, and then subjected to immunoblotting against ASK1 (top). Cotransfection of ASK1t and the human ASK1 (hASK1) restores the expression level of ASK1 in cells (bottom). B, Knockdown of ASK1 expression in MN9D cells prevents the activation of JNK (p-JNK), c-Jun (p-c-Jun), and p38 (p-p38) at 2 and 8 hafter 6-0HDA (50 $\mu$ m) exposure. $\boldsymbol{C}$, Quantitative results of (B). ${ }^{*} p<$ $0.05,{ }^{* *} p<0.01$ between the groups indicated; data are from four experiments. $\boldsymbol{D}-\boldsymbol{F}$, Knockdown of ASK1 expression is neuroprotective against 6-0HDA neurotoxicity in MN9D cells ( $\boldsymbol{D}$ ); cotransfection of hASK1 restores the suppressed (by ASK1t) 6-OHDA neurotoxicity $(\boldsymbol{E})$; overexpression of ASK1dn promotes cell survival after 6-OHDA neurotoxicity $(\boldsymbol{F})$. In all experiments described in $\boldsymbol{D}-\boldsymbol{F}$, cell viability was measured at $24 \mathrm{~h}$ after $6-0 \mathrm{HDA}$ ( 50 and $100 \mu \mathrm{m}$ ) exposure. ${ }^{*} p<0.05,{ }^{* *} p<0.01$ between the groups indicated; $n=12$ from three to four independent experiments.

6-OHDA-induced phosphorylation of c-Jun, a downstream target of JNK and a sensitive marker for JNK activation (Fig. $4 E$ ).

One of the upstream stress-related kinase activators for both the JNK and p38 pathways in neuronal cells is ASK1 (Takeda et al., 2004; Ouyang and Shen, 2006; Stetler et al., 2008). We further showed that exposure of MN9D cells to 6-OHDA produced a dramatic and persistent increase in ASK1 phosphorylation (Fig. $4 C$ ), and this increase could be inhibited by PRX2 overexpression (Fig. $4 D, E$ ). ASK1 activity measured by a protein kinase assay was also increased after 6-OHDA treatment, and was nearly completely inhibited by PRX2 overexpression (Fig. 4D). In contrast, knockdown of PRX2 expression significantly enhanced the activation of ASK1 (data not shown). These data indicate that PRX2 inhibited 6-OHDA-induced activation of ASK1 and the downstream JNK and p38 pro-death signaling pathways in DA neurons.

\section{PRX2 inhibits the activation of ASK1 by interfering with its dissociation from Trx}

We next investigated how PRX2 prevents the activation of ASK1 following 6-OHDA neurotoxicity. Under unstressed conditions, ASK1 is known to form a signalosome bound to its negative regulator, Trx. Upon oxidation by cellular oxidants, Trx dissociates from ASK1, resulting in the activation of ASK1 and subsequent cell death via the JNK and p38 pathways (Fujino et al., 2007). The known mammalian PRXs, with the exception of PRX4, use Trx as an electron donor to reduce oxidized PRXs back into their functional and protective state (Low et al., 2008). We speculated that Trx might be the bridging point through which PRX2 regulates ASK1 activation. To test this hypothesis, we first took advantage of an antibody that recognized both oxidized (higher molecular weight) and reduced (lower molecular weight) Trx to examine the effect of 6-OHDA on the redox status of Trx. As shown in Figure $5 A$, an increase in oxidized Trx was seen as early as $1 \mathrm{~h}$ after 6-OHDA exposure and reached peak levels at 8-16 h. In contrast, the levels of reduced Trx were decreased at 2-16 h after 6-OHDA exposure (Fig. 5A). Consistent with the role of PRX2 as a negative regulator for Trx oxidation, lentivirusmediated overexpression of PRX2 prevented the accumulation of oxidized Trx after 6-OHDA exposure (Fig. 5B), whereas expression of a PRX2dn, in which both conserved Cys residues (amino acids 51 and 172) were replaced with alanine, enhanced the oxidation of Trx (Fig. 5C). Together with previous result showing that PRX2 overexpression resulted in a significant increase in PRX- $\mathrm{SO}_{3}$ levels (Fig. 2J), the robust inhibitory effect of PRX2 overexpression on the formation of oxidized Trx following 6-OHDA neurotoxicity suggests that the ratio between cellular reduced (active) and oxidized (inactive) PRXs, but not the absolute amount of oxidized PRXs, is a key determinant for the redox state of Trx. Supporting this notion, PRX2 transfection in MN9D cells with or without 6-OHDA exposure resulted in remarkable increases in total cellular PRX activity (Fig. 2C), an index for PRX antioxidant (reduced) reserves. Indeed, assessment of $\mathrm{H}_{2} \mathrm{O}_{2}$ levels using the DCF (dichlorofluorescein) fluorescence assay 
A

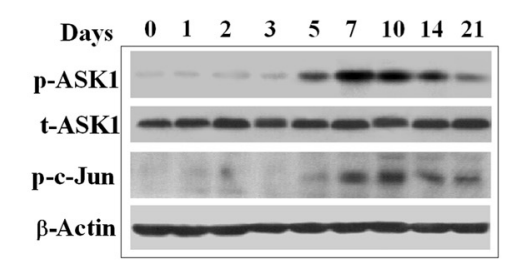

B

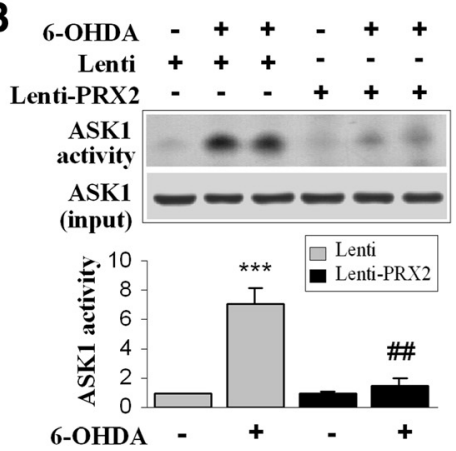

E
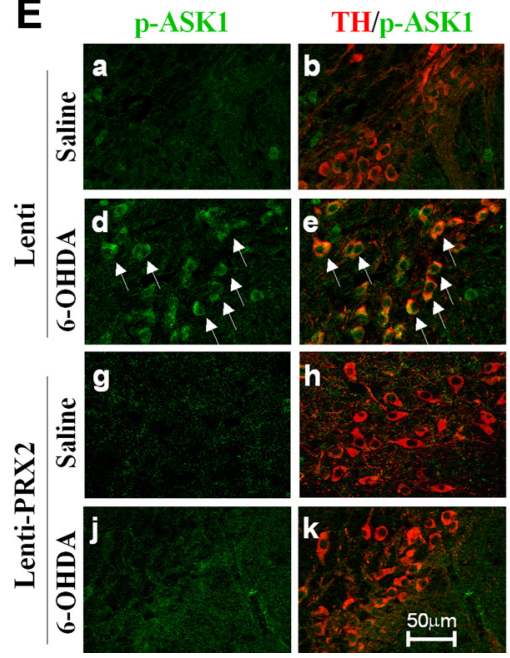

C

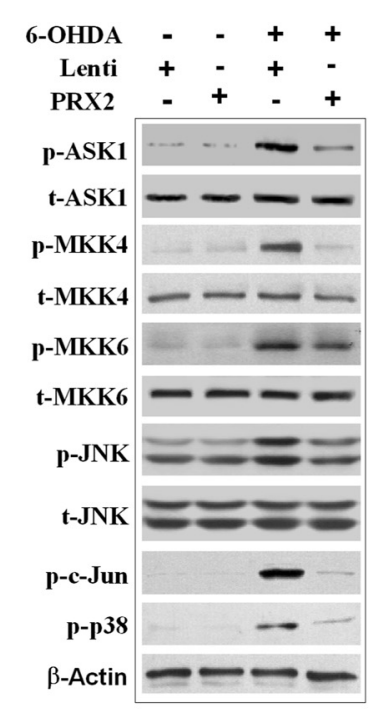

D
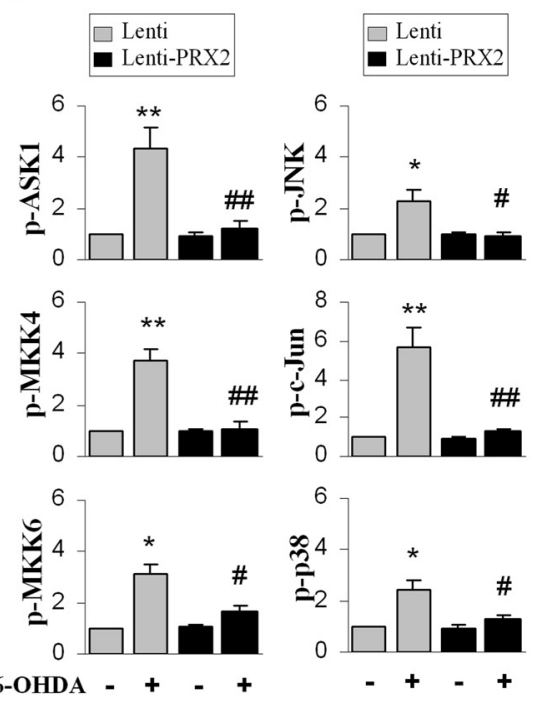

6-OHDA - + - +

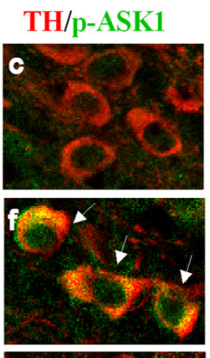

$\mathbf{F}$
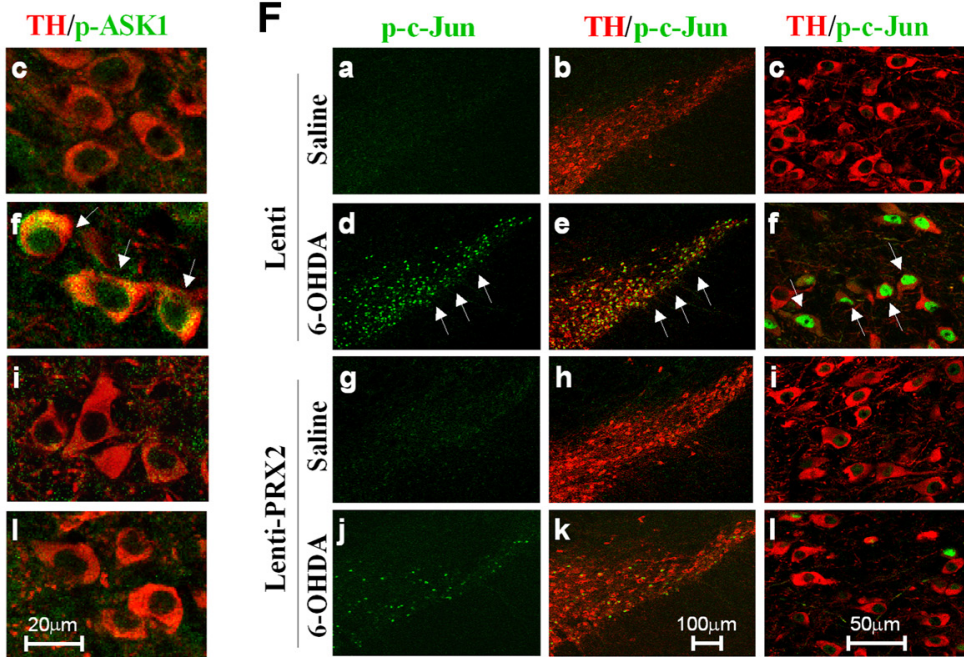

Figure 7. PRX2 suppresses the ASK1 signaling cascade in 6-OHDA-lesioned mice. A,6-OHDA lesioning activates ASK1 signaling in mouse SNc. Mice were subjected to striatal infusion of 6-OHDA (3.0 $\mu \mathrm{g})$, and at the indicated time points the SNc extracts were processed for immunoblotting against p-ASK1 and p-c-Jun, a sensitive marker for JNK activation. The blots are representative of three experiments with similar results. $\boldsymbol{B}-\boldsymbol{D}$, PRX2 inhibits the ASK1 signaling cascade in 6-OHDA-lesioned mice. Mouse SNc was infected for $21 \mathrm{~d}$ with empty lentiviral vector (Lenti) or Lenti-PRX2, and then received striatal infusion of 6-0HDA $(3.0 \mu \mathrm{g})$ or saline. At $7 \mathrm{~d}$ after 6-OHDA infusion, SNc extracts were subjected to ASK1 kinase activity assay ( $\boldsymbol{B}$ ) and immunoblotting against p-ASK1 and its downstream signaling proteins ( $(\boldsymbol{C}$. Quantitative data areillustrated in $\boldsymbol{D}$. ${ }^{*} p<0.05$, ${ }^{* *} p<0.01$ versus vehicle control; $\# p<0.05$, \#\#p $<0.01$ versus empty lentivirus group; $n=4 /$ group. E, Double-label immunofluoroscent staining forpASK1 (green) and TH (red) in the SNc 21 dafter brain inoculation with either empty lentiviral vector $(\boldsymbol{a}, \boldsymbol{b}, \boldsymbol{d}, \boldsymbol{e}$, low magnification; $\boldsymbol{c}, \boldsymbol{f}$, high magnification) or Lenti-PRX2 ( $\boldsymbol{g}, \boldsymbol{h}, \boldsymbol{j}, \boldsymbol{k}$, low magnification; $\boldsymbol{i}, \boldsymbol{l}$, , high magnification) and $7 \mathrm{~d}$ after saline $(\boldsymbol{a}-\boldsymbol{c}, \boldsymbol{g}-\boldsymbol{i})$ or 6-OHDA $(\boldsymbol{d}-\boldsymbol{f}, \boldsymbol{j}-\boldsymbol{l})$ infusion. Note that increased p-ASK1 shows mainly a cytosolic localization after 6-OHDA lesioning, and p-ASK1 is attenuated in Lenti-PRX2-infected SNc. Scale bars: low magnification, $50 \mu \mathrm{m}$; high magnification, $20 \mu \mathrm{m}$. $\boldsymbol{F}$, Double-label immunofluoroscent staining for p-c-Jun (green) and TH (red) in the SNc $21 \mathrm{~d}$ after brain inoculation with either empty lentiviral vector ( $\boldsymbol{a}, \boldsymbol{b}$, $\boldsymbol{d}, \boldsymbol{e}$, low magnification; $\boldsymbol{c}, \boldsymbol{f}$, high magnification) or Lenti-PRX2 $(\boldsymbol{g}, \boldsymbol{h}, \boldsymbol{j}, \boldsymbol{k}$, low magnification; $\boldsymbol{i}, \boldsymbol{l}$, , high magnification) and $7 \mathrm{~d}$ fter saline $(\boldsymbol{a}-\boldsymbol{c}, \boldsymbol{g}-\boldsymbol{i})$ or $6-0 \mathrm{HDA}(\boldsymbol{d}-\boldsymbol{f}, \boldsymbol{j}-\boldsymbol{l})$ infusion. Note that increased $p--(-J u n$ shows mainly a nuclear localization after 6-OHDA lesioning, and p-ASK1 is attenuated in Lenti-PRX2-infected SNc. Scale bars: low magnification, $100 \mu \mathrm{m}$; high magnification, $50 \mu \mathrm{m}$.

showed that PRX2 overexpression shifted the intracellular environment of MN9D cells from an oxidized state to a reduced state under 6-OHDA neurotoxicity, whereas PRX2 knockdown promoted the oxidized state (supplemental Fig. 4, available at www. jneurosci.org as supplemental material).

We then showed that PRX2 inhibits the dissociation of Trx and ASK1. MN9D cells were collected 2 and $8 \mathrm{~h}$ after 6-OHDA or vehicle treatment. Whole-cell lysates were subjected to IP with an ASK1-specific antibody, followed by immunoblotting of the precipitates against Trx. Under control conditions, a co-IP of Trx and ASK1 was readily detectable (Fig. 5D). Treatment with 6-OHDA resulted in the dissociation of Trx from ASK1, leading to decreased Trx density in the ASK1 precipitates. PRX2 over- expression prevented the dissociation between Trx and ASK1 following 6-OHDA treatment (Fig. $5 D, E$ ). Reverse co-IP confirmed the effect of PRX2 on the association between ASK1 and $\operatorname{Trx}$ (Fig. 5D).

\section{ASK1 is a critical mediator of DA neuronal death following 6-OHDA in vitro}

To further characterize the functional role of ASK1 in 6-OHDAinduced DA cell death, MN9D cells were transfected for $3 \mathrm{~d}$ with lentiviral vectors expressing either an ASK1-targeting shRNA (Lenti-ASK1t) or a scrambled control sequence (Lenti-ASK1sc). Transfection of Lenti-ASK1t but not Lenti-ASK1sc or the GFP control vector reduced ASK1 expression in MN9D cells by $>90 \%$ 
A

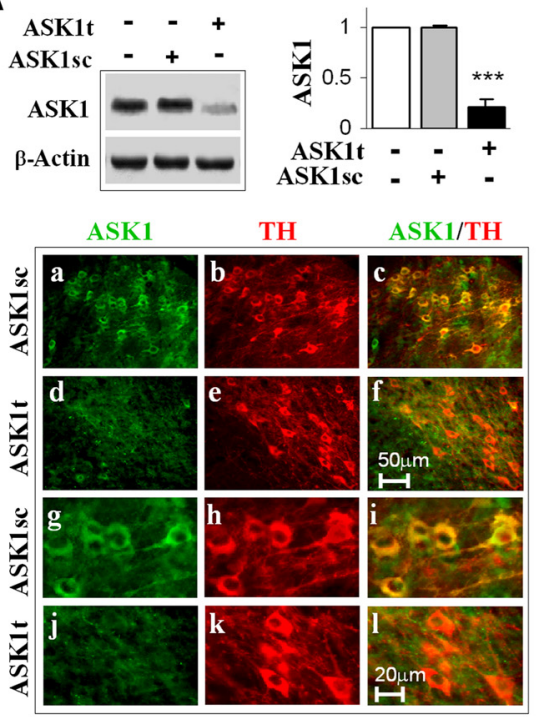

D

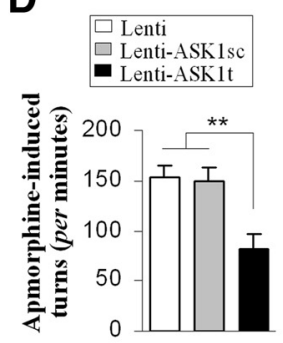

$\mathbf{E}$

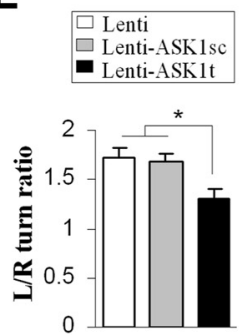

B

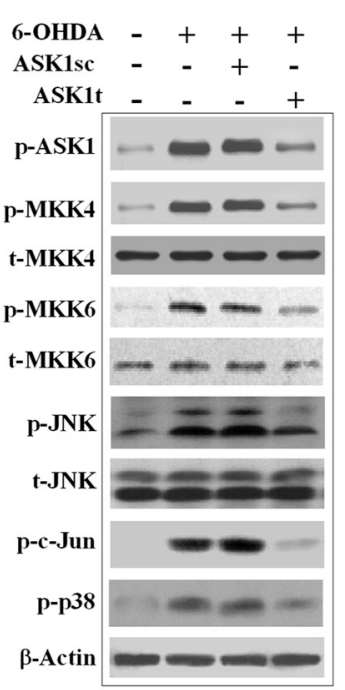

C
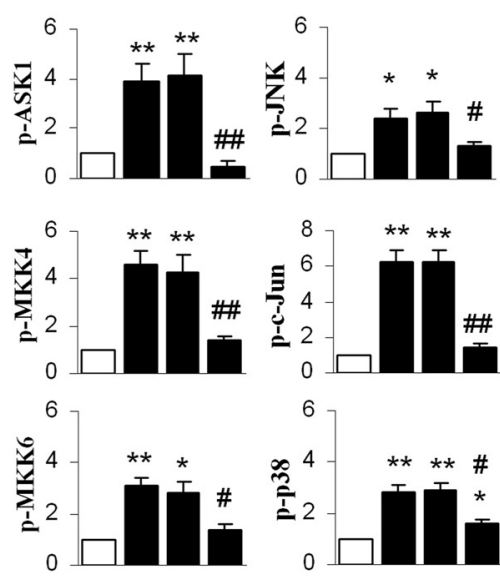

6-OHDA - +++

ASK1sc - - + -

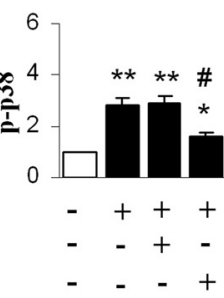

$\mathbf{F}$

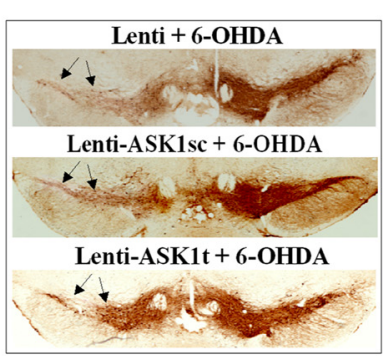

G

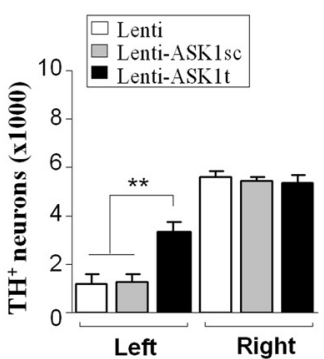

H
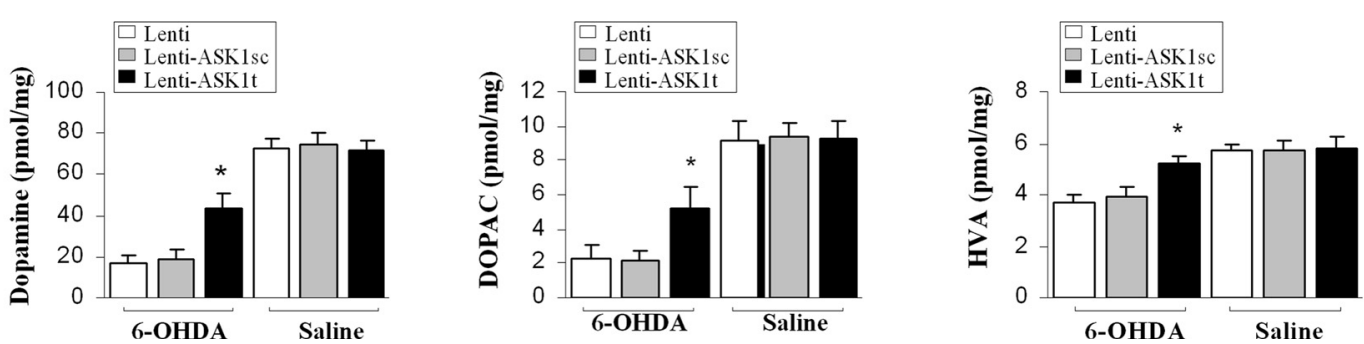

Figure 8. ASK1 activation contributes to nigrostriatal neurodegeneration in 6-OHDA-lesioned mice. A, Lentivirus-mediated knockdown of ASK1 expression in mouse SNc. Mouse SNc was infected for $21 \mathrm{~d}$ with empty lentiviral vector, Lenti-ASK1t, or Lenti-ASK1sc, and then subjected to immunoblotting against ASK1 and double-label immunofluorescent staining for ASK1 and TH. ${ }^{* * *} p<$ 0.001 versus empty vector control; $n=5$ /group. Scale bars: $\boldsymbol{a}-\boldsymbol{f}$ (low magnification), $50 \mu \mathrm{m} ; \boldsymbol{g}-\boldsymbol{I}$ (high magnification), $20 \mu \mathrm{m}$. $\boldsymbol{B}, \boldsymbol{C}$, ASK1 knockdown attenuates the ASK1 signaling cascade in 6-OHDA-lesioned mice. Mouse SNc was infected for $21 \mathrm{~d}$ with the lentiviral vectors described in $\boldsymbol{A}$, and the mice received striatal infusion of 6-0HDA (3.0 $\mu \mathrm{g}$ ) or saline. $\boldsymbol{B}, \boldsymbol{C}$, Immunoblotting against p-ASK1 and its downstream signaling proteins was performed $7 \mathrm{~d}$ after $6-0 \mathrm{HDA}$ infusion $(\boldsymbol{B})$; quantitative data of $\boldsymbol{B}$ are illustrated in $\boldsymbol{C}$. ${ }^{*} p<0.05,{ }^{* *} p<0.01$ versus vehicle control; \#p $<0.05$, $\# \# p<0.01$ versus empty lentivirus group; $n=5-6 /$ group. $D-G$, ASK1 knockdown attenuates DA neuron loss and neurological deficits in 6-0HDA-lesioned mice. Mice were treated as described in $\boldsymbol{A}$; behavioral tests $(\boldsymbol{D}, \boldsymbol{E})$ and histology $(\boldsymbol{F}, \boldsymbol{G})$ were performed $21 \mathrm{~d}$ after 6-0HDA infusion. Apomorphine-induced circling after 6-0HDA injection is shown as the number of turns in the first 30 min after intraperitoneal injection of apomorphine (D). The corner test shows the mean of the ratio of spontaneous left/right turns of 10 trials/session $21 \mathrm{~d}$ after 6 - 0 HDA injection ( $\boldsymbol{E}$ ). Arrows in $\boldsymbol{F}$ indicate the SNc region lesioned by 6-OHDA. The numbers of TH-positive neurons in the SNc of three experimental groups are illustrated in $\mathbf{G}$. $\boldsymbol{H}$, Catecholamine levels from the ipsilateral striatum $21 \mathrm{~d}$ following the indicated injections. Catecholamines are expressed as picomoles/milligram fresh, wet tissue. ${ }^{*} p<0.05,{ }^{* *} p<0.01$ between the groups indicated; $n=10-12$ per group for all experiments.

(Fig. 6A,B). Targeted ASK1 knockdown abolished 6-OHDAinduced phosphorylation of ASK1, JNK, and p38 (Fig. 6B,C), and significantly increased cell survival following 6-OHDA (Fig. 6D). Coinfection of Lenti-ASK1t with human ASK1 (Lenti-ASK1 $\mathrm{h}$ ), which is resistant to shRNA targeting the mouse ASK1, prevented the neuroprotective effect of Lenti-ASK1t (Fig. 6E). These results thus confirmed the neuroprotective effect of ASK1 knockdown against 6-OHDA neurotoxicity. In another set of experiments, lentiviral transfection of $\mathrm{ASK} 1_{\mathrm{K} 709 \mathrm{R}}$, a dominant-negative mutant of ASK1 (ASK1dn) in MN9D cells, was also neuroprotective against 6-OHDA-induced cell death (Fig. $6 F$ ). Together, the data presented here support a critical role for ASK1 in 6-OHDAinduced DA neuronal death.

PRX2 protects against DA neurodegeneration via inhibiting the activation of the ASK1-JNK/p38 signaling cascade in 6-OHDA-lesioned mice

In view of the importance of ASK1 and the JNK and p38 signaling pathways for both 6-OHDA-induced DA cell death and PRX2 neuroprotection in vitro, we further investigated the role of this oxidative stress-responsive pro-death signaling cascade in the in vivo 6-OHDA model of PD. The phosphorylation of ASK1 was 
increased in the $\mathrm{SNc}$ beginning at $5 \mathrm{~d}$, peaking at 7-10 $\mathrm{d}$ and remaining elevated at $21 \mathrm{~d}$ after 6-OHDA lesioning (Fig. 7A). The phosphorylation of c-Jun was also robustly increased after 6-OHDA lesioning (Fig. 7A).

To confirm that PRX2 overexpression conferred neuroprotection through the inhibition of ASK1 signaling, we first determined whether PRX2 overexpression was able to inhibit ASK1 activation $7 \mathrm{~d}$ after 6-OHDA insult, when maximal activation of ASK1 was seen. Indeed, PRX2 overexpression led to a significant inhibition of 6-OHDA-induced ASK1 kinase activity (Fig. $7 B$ ). The phosphorylation status of ASK1 and its downstream signaling molecules were also examined in $\mathrm{SNc}$ $7 \mathrm{~d}$ after 6-OHDA lesioning using Western blot analysis (Fig. 7C). Consistent with our in vitro observation, 6-OHDA lesioning increased the levels of phospho-ASK1, phospho-JNK, phospho-c-Jun, and phospho-p38 in SNc. The phosphorylation of MKK4 and MKK6, two enzymes important in mediating ASK1induced activation of JNK and p38 pathways, respectively, were also elevated in $\mathrm{SNc}$ by 6-OHDA lesioning. Lentivirusmediated overexpression of PRX2 inhibited 6-OHDA-induced phosphorylation of ASK1, MKK4, MKK6, c-Jun, JNK, and p38 (Fig. 7C,D). Double-immunofluorescent staining revealed that phosphorylated ASK1 (Fig. 7E) and phosphorylated c-Jun (Fig. $7 F$ ) were increased dramatically in TH-positive DA neurons on the ipsilateral side of the 6-OHDA-lesioned $\mathrm{SNc}$. The neuronal expression of phosphorylated ASK1 and phosphorylated c-Jun was largely blocked by Lenti-PRX2 compared with that of empty vectortreated animals. These results thus support the hypothesis that PRX2 inhibits ASK1 activation and subsequent JNK and p38 pro-death signaling pathways in 6-OHDA-lesioned mice.

\section{Inhibition of ASK1 protects DA} neurons from 6-OHDA neurotoxicity in vivo

Our in vitro findings suggested that ASK1 activation is a critical signaling event governing 6-OHDA-induced degeneration of DA neurons; therefore, we further evaluated the potential protective effect of blocking ASK1 activity in vivo. Lenti-ASK1t or Lenti-ASK1sc was infused into the left SNc 3 weeks before 6-OHDA lesioning. Determined at 3 weeks after shRNA delivery, Lenti-ASK1t knocked down ASK1 expression to $~ 20 \%$ of control levels, while ASK1sc had no effect on ASK1 expression (Fig. 8A). Double labeling using ASK1 and TH antibodies revealed a loss of ASK1 expression in TH-positive DA neurons in the SNc of ASK1t, but not in
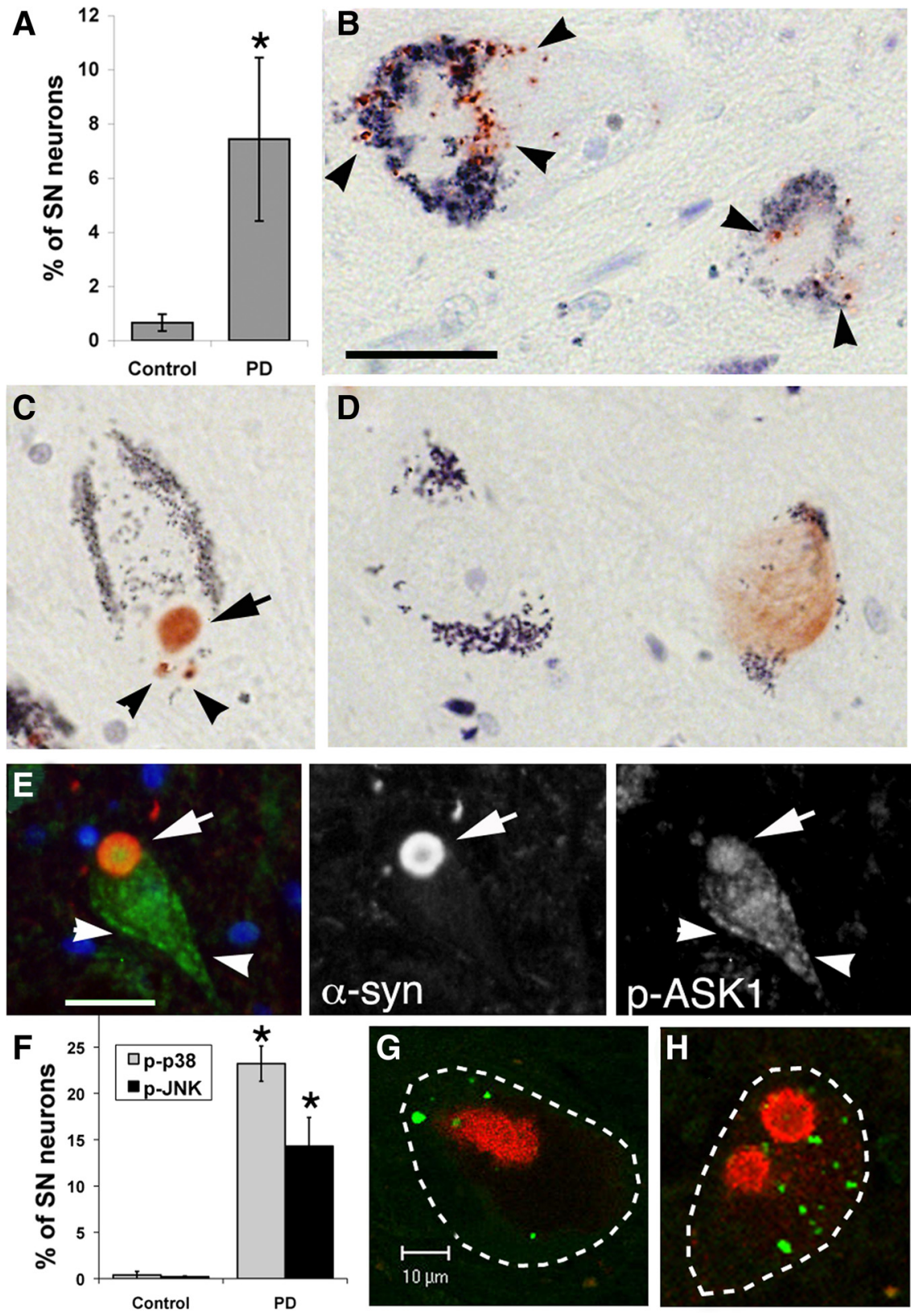

Figure 9. Activation of p-ASK1, p-JNK, and p-p38 pathways in SNc neurons of sporadic PD patients. $\boldsymbol{A}-\boldsymbol{E}, \mathrm{p}$-ASK1 staining in the SNc neurons of human brains. The SNc neurons of PD patients exhibited increased staining for P-ASK1 compared with control subjects $(\boldsymbol{A})$. In PD patients, the most common staining pattern of p-ASK1 was a granular, cytoplasmic distribution ( $\boldsymbol{B}$, arrowheads). In 33\% of cases, p-ASK1 staining was colocalized with Lewy body/pale body staining ( $\boldsymbol{C}$, arrow). In normal control subjects and in disease specificity controls with pure Alzheimer's disease, a few neurons exhibiting p-ASK1 immunoreactivity typically showed a light diffuse staining pattern ( $\boldsymbol{D}$, right cell), and $99.4 \%$ of neurons were negative ( $\boldsymbol{D}$, left cell). Double-label immunofluorescence confirmed colocalization of $\alpha$-synuclein (red) with p-ASK1 (green) in the Lewy body (arrow), on a background of granular cytoplasmic p-ASK1 staining ( $\boldsymbol{E}$, arrowheads). $\boldsymbol{F}-\boldsymbol{H}, \mathrm{p}$-p38 and p-JNK staining in the SNc neurons of human brains. Significantly more SNc neurons from PD patients exhibited immunoreactivity for p-p38 and for p-JNK $(\boldsymbol{F})$. While p-p38 and p-JNK staining was not colocalized with $\alpha$-synuclein, $>70 \%$ of neurons with granular p-p38 (G, green) or p-JNK ( $\boldsymbol{H}$, green) staining also showed abnormal $\alpha$-synuclein aggregation (red). The outline of the individual neuron is indicated by dashes. Scale bars: $\boldsymbol{B}-\boldsymbol{D}, 25 \mu \mathrm{m} ; \boldsymbol{E}-\boldsymbol{H}, 10$ $\mu \mathrm{m} .{ }^{*} p<0.05$ versus control.

ASK1sc-infected mice (Fig. 8A). The phosphorylation status of ASK1 and its downstream signaling molecules were examined in Lenti-ASK1t- or Lenti-ASK1sc-infected and noninfected animals at $7 \mathrm{~d}$ after 6-OHDA lesioning. Lentivirus-mediated expression of ASK1t, but not ASK1sc shRNA, significantly attenuated 6-OHDA-induced phosphorylation of ASK1, MKK4, MKK6, c-Jun, JNK, and p38 (Fig. 8B,C). These results confirmed our 
findings in cell cultures that ASK1 is essential for 6-OHDAinduced activation of JNK and $\mathrm{p} 38$ pro-death signaling pathways. The inhibition of ASK1 by Lenti-ASK1t protected against 6-OHDA-induced death of TH-positive DA neurons in SNc (Fig. $8 F, G)$ improved motor functions compared with Lenti-ASK1scinfected animals (Fig. 8D,E), and significantly elevated the concentrations of DA and its metabolites in the striatum (Fig. $8 \mathrm{H}$ ).

\section{Activation of ASK1 and downstream JNK/p38 signaling is observed in degenerating human PD SNc neurons}

Our in vitro and in vivo animal data demonstrated the importance of the ASK1-JNK/p38 signaling pathway in DA neuronal apoptosis in response to a PD-related neurotoxin and suggested the therapeutic potential by blocking this pathway. To confirm whether or not these cell death pathways are activated in PD, we analyzed human postmortem midbrain sections from normal controls, $\mathrm{AD}$ patients (as a disease specificity control), and $\mathrm{PD}$ patients for expression of phosphorylated ASK1, JNK, and p38 MAPK. SNc neurons from PD patients showed significantly increased staining for p-ASK1 compared with SNc neurons from either normal subjects or AD patients (Fig. $9 A-D$ ). In $\mathrm{PD}$ patients, p-ASK1 most frequently exhibited a punctate cytoplasmic staining pattern (Fig. 9A), although colocalization with Lewy bodies was observed in $33 \%$ of the cases (Fig. 9B,E). There were significantly more $\mathrm{SNc}$ neurons exhibiting positive staining for p-JNK and p-p38 in PD compared with control groups, exhibiting predominantly a punctate cytoplasmic distribution (Fig. $9 F-$ $H)$. Double-immunofluorescence labeling for $\alpha$-synuclein and each phosphokinase confirmed Lewy body colocalization for p-ASK1 (Fig. 9E), but not for p-JNK and p-p38 (Fig. 9G,H). However, $>70 \%$ of individual neurons staining for $\mathrm{p}-\mathrm{JNK}$ and p-p38 and $>60 \%$ of neurons with p-ASK 1 also showed abnormal $\alpha$-synuclein staining, indicating activation of these cell death signaling pathways in degenerating SNc neurons in PD.

\section{Discussion}

Although a number of genes and environmental factors have been identified as causes of PD, most are of unknown etiology (Farrer, 2006). An improved understanding of the mechanism for the DA cell death/survival common to all forms of the disease is therefore essential for developing broadly applicable treatment strategies. This study demonstrates that, in addition to its commonly recognized role as an antioxidant enzyme, PRX2 plays a critical role in protecting DA neurons from PD-relevant toxininduced cell death through regulating Trx-ASK1 interactions and inhibiting subsequent activation of ASK1-dependent cell death pathways. We demonstrate here that PRX2 overexpression or ASK1 knockdown significantly protects DA neurons from 6-OHDA toxicity, preserving motor functions involving the dopamine system.

Identification of PRX2 as an important PRX in DA neuronal survival is particularly relevant since PRX2 localizes almost exclusively to neurons and is the most abundant PRX enzyme in mammalian neurons (Jin et al., 2005). Moreover, PRX2 is abundantly expressed in murine brain cells that use oxygen at high rates (Sarafian et al., 1999; Jin et al., 2005). DA neurons are notorious for their vulnerability to oxidative stress, partly due to their higher rate of oxygen metabolism. Altered expression of $\mathrm{PRX} 2$ has been reported in the brains of PD patients, supporting the clinical relevance of this neuron-specific PRX (Krapfenbauer et al., 2003; Basso et al., 2004; Fang et al., 2007; Qu et al., 2007).

Although the current study suggests that PRX2 may be the most important endogenous 2-Cys PRX for preventing apoptosis

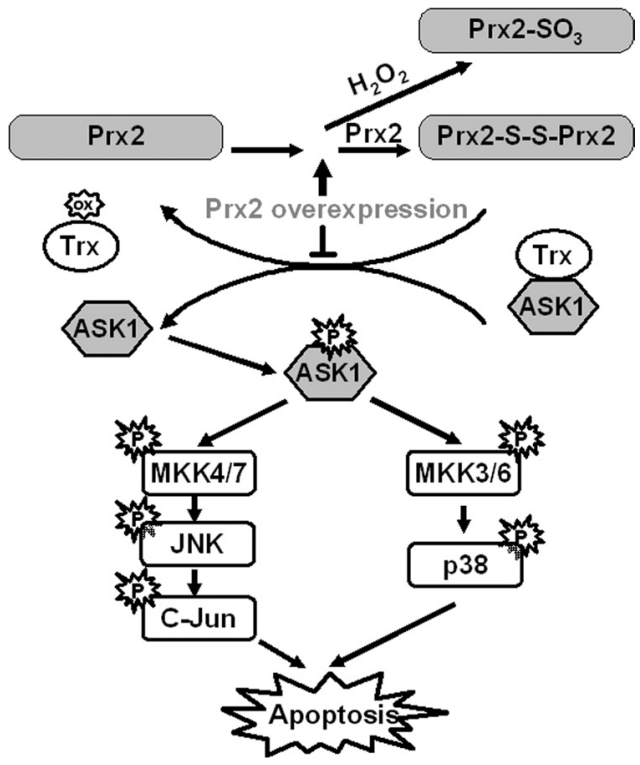

Figure 10. Proposed pathways for PRX2 overexpression conferred neuroprotection against 6-OHDA. Based on our findings, under oxidative stress induced by 6-OHDA, PRXs are readily oxidized with a consequent reduction in antioxidative activity. Trx was oxidized in an effort to reduce the oxidized PRX. Oxidized Trx then dissociated from ASK1, leading to the activation of ASK1 and subsequent p38 and JNK apoptotic cascades. Replenishment of abundant amounts of functional deoxidized PRX2 inhibit the cysteine thiol-disulfide exchange between PRX2 and Trx and drive the PRX-Trx reaction toward the production of overoxidized PRXs, resulting in the reduction of oxidized Trx and an increase in $\mathrm{PRX}-\mathrm{SO}_{3}$ after $\mathrm{PRX} 2$ overexpression. As a consequence, more reduced Trx is available to inhibit the activation of ASK1 and related cell death events.

due to 6-OHDA challenge, it does not exclude the potential role of other 2-Cys PRXs in protecting against the loss of DA neurons. In fact, our results from MN9D cells showed that PRX1 overexpression has a protective effect against DA cell death similar to that of PRX2, although knockdown of endogenous PRX2 shows a greater impact on cell viability than does PRX1. The recent report by Lee et al. (2008) also documented the protective effect of PRX1 in a cellular model of 6-OHDA toxicity. It is noteworthy that PRX1 mainly expresses in glial cells in the brain; the protective effect of PRX1 thus might be significant in other neurotoxin PD models with direct glial involvement, such as lipopolysaccharide or MPTP (1-methyl-4-phenyl-1,2,3,6-tetrahydropyridine) models. PRX4 was also protective against DA cell death. However, PRX4 knockdown resulted in only marginal reduction in total cellular PRX activity, suggesting that PRX4 activity may be redundant in DA cells and that its loss can be compensated for by other PRXs. PRX3, a mitochondrial-specific PRX, failed to confer significant neuroprotection against DA cell death induced by 6-OHDA. This result, however, may be model specific, since 6-OHDA, instead of directly targeting mitochondria, initiated its toxicity via extracellular auto-oxidation and the induction of oxidative stress from the oxidative products generated (Hanrott et al., 2006). Future studies are warranted to investigate the effect of PRX3 in PD models with direct mitochondria-targeting neurotoxins such as MPTP or rotenone. In fact, PRX3-depleted SH-SY5Y cells have been shown to be more sensitive to $\mathrm{MPP}^{+}$induced cell death (De Simoni et al., 2008).

It has been reported previously that overoxidized PRX1 (PRX1-SO $\mathrm{S}_{2}$ and PRX1-SO $\mathrm{S}_{3}$ ) is increased in PD models (Lee et al., 2008). The present study detected increased levels of total PRX$\mathrm{SO}_{3}$ in both MN9D DA neurons and in mouse $\mathrm{SNc}$ in response to 6-OHDA lesioning, and it appears that $\mathrm{PRX} 2-\mathrm{SO}_{3}$ accounts for a 
larger portion of the total $\mathrm{PRX}-\mathrm{SO}_{3}$ increases. The increased $\mathrm{PRX}-\mathrm{SO}_{3}$ was accompanied by remarkably reduced cellular PRX activity. These results support the notion that the neuron-specific PRX2 is the predominant PRX involved in reactions to 6-OHDA toxicity, where PRX2 becomes overoxidized while scavenging peroxides, resulting in the depletion of cellular PRX activity reserves. Several other modifications of PRX2, including phosphorylation and nitrosylation, have been observed in the brain of PD patients and are thought to be the cause of loss of function for PRX2 (Fang et al., 2007; Qu et al., 2007). The results presented here suggest that oxidation is another debilitating modification that could diminish the protective capacity of PRX2 in PD. It is known that overoxidized PRX2, although resistant to reduction by Trx, can be reduced and reactivated by sulforedoxin. However, the renewal of overoxidized PRX is extremely slow in mammalian cells (Rhee et al., 2005). The slow reactivation of PRX2 may explain why overexpression of PRX2 in the midbrain not only replenished the deoxidized PRX2, allowing functional PRXs to join the defense against 6-OHDA-induced oxidative stress, but also increased levels of oxidized PRXs. Large amounts of deoxidized PRX2 may also inhibit the enzymes responsible for PRX deoxidation, such as Trx and sulforedoxin, resulting in increased accumulation of $\mathrm{PRX}-\mathrm{SO}_{3}$. Thus, our data suggest that PRX2 overoxidation is a pathological change indicating a disturbance of the PRX system in PD. However, the increased amount of overoxidized PRX does not necessarily parallel neuronal death, particularly after PRX replenishment, and the ratio of reduced PRX to oxidized PRX may be a better predictor for DA neuronal survival.

The mechanism by which PRX2 protects neurons has largely been attributed to its antioxidative properties. The present study demonstrated that in addition to rescuing PRX enzyme activity, PRX2 also shows antiapoptotic effects in DA neurons manifested by its inhibition of the caspase-3- and caspase-9-dependent intrinsic apoptotic pathway following 6-OHDA treatment. In line with our observation, PRX2 has been shown to inhibit caspase activation in cardiomyocytes (Zhao et al., 2009). We further extend our findings by demonstrating that PRX2 inhibited the upstream stress-response kinase ASK1. Many toxin-induced models of PD induce ASK1, including MPTP, 6-OHDA, and paraquat (Ouyang and Shen, 2006; Karunakaran et al., 2007; Niso-Santano et al., 2010). In support for an important role for ASK1 in neuronal apoptosis, primary neurons derived from ASK1 knock-out mice exhibit a remarkable resistance to JNK and p38 activation and to apoptosis induced by oxidative or endoplasmic reticulum (ER) stress (Kadowaki et al., 2005; Harada et al., 2006). Our data show that PRX2 protected DA neurons by inhibiting ASK1 activity and the subsequent activation of JNK/cJun and p38 cell death pathways. As degenerating SNc neurons from PD patients show evidence of ASK1, JNK, and p38 pathway activation, the protective effects of PRX2 might be extrapolated to include sporadic PD as well as other ASK-dependent stressors, such as ER stress, DNA damage, and inflammation.

Another important finding in this study was in identifying a mechanistic link between PRX, Trx and ASK1, and how an oxidative load leads to apoptotic cell death (Fig. 10). Trx has at least two known physiological functions. First, reduced Trx offers electrons to regenerate active PRX; second, reduced Trx binds to ASK1 and inhibits its kinase activity. Based on our findings, under oxidative stress such as that induced by 6-OHDA, Trx was oxidized in an effort to reduce the oxidized PRX. Oxidized Trx then dissociated from ASK1, leading to the activation of ASK1 and subsequent apoptotic cascades. Replenishment of abundant amounts of functional deoxidized PRX2 might inhibit the cysteine thiol-disulfide exchange between PRX2 and Trx, and drive the PRX-Trx reaction toward the production of overoxidized PRXs, resulting in the reduction of oxidized Trx and a significant increase in $\mathrm{PRX}-\mathrm{SO}_{3}$ after PRX2 overexpression. As a consequence, more reduced Trx is available to inhibit the activation of ASK1 and related cell death events.

Given that the signal transduction from PRX2 to ASK1 via Trx could effectively respond to oxidative stress and activate apoptotic machinery, it is conceivable that any step in this cascade could be a legitimate target for anti-death intervention in oxidative stress-involved diseases. In support of this notion, we demonstrated that both PRX2 overexpression and ASK1 knockdown could significantly protect DA neurons from 6-OHDA toxicity and improve motor function. While PRX2 is expressed almost exclusively in neurons, ASK1 is ubiquitously expressed in nearly all types of cells in the brain, including glial cells (Faigle et al., 2004). Recent publications have established a role of ASK1 in inflammatory responses (Liu et al., 2006; Kanayama and Miyamoto, 2007; Yang et al., 2008). Our Lenti-ASK1t construction under the control of U6 promoter allowed for expression in all types of brain cells. Thus, it is possible that ASK1 knockdown not only acted directly on DA neurons, but also reduced cerebral inflammation in response to the neurotoxin 6-OHDA. Neuroinflammation contributes to the progression of PD (Block et al., 2007). Thus, although Lenti-ASK1t demonstrated DA system protection almost comparable to that from neuronal PRX2 overexpression within our observation period (3 weeks after 6-OHDA), it might afford significant long-term protection in experimental PD models or in PD, due to its potential suppression of neuroinflammation.

In summary, the observations presented in this study describe a previously undefined cell death mechanism in DA neurons by which the redox-sensitive molecules PRX2 and Trx are able to modulate apoptotic pathways (ASK1, MKKs, JNK, and p38) in response to a $\mathrm{PD}$-related toxic stress. Our results suggest that PRX2 and ASK1 may be promising and novel targets for neuroprotective intervention in PD.

\section{References}

Bae JY, Ahn SJ, Han W, Noh DY (2007) Peroxiredoxin I and II inhibit $\mathrm{H} 2 \mathrm{O} 2$-induced cell death in MCF-7 cell lines. J Cell Biochem 101:1038-1045.

Basso M, Giraudo S, Corpillo D, Bergamasco B, Lopiano L, Fasano M (2004) Proteome analysis of human substantia nigra in Parkinson's disease. Proteomics 4:3943-3952.

Block ML, Zecca L, Hong JS (2007) Microglia-mediated neurotoxicity: uncovering the molecular mechanisms. Nat Rev Neurosci 8:57-69.

Braak H, Braak E (1991) Neuropathological stageing of Alzheimer-related changes. Acta Neuropathol 82:239-259.

Braak H, Del Tredici K, Rüb U, de Vos RA, Jansen Steur EN, Braak E (2003) Staging of brain pathology related to sporadic Parkinson's disease. Neurobiol Aging 24:197-211.

Cao G, Pei W, Ge H, Liang Q, Luo Y, Sharp FR, Lu A, Ran R, Graham SH, Chen J (2002) In vivo delivery of a Bcl-xL fusion protein containing the TAT protein transduction domain protects against ischemic brain injury and neuronal apoptosis. J Neurosci 22:5423-5431.

Choi WS, Eom DS, Han BS, Kim WK, Han BH, Choi EJ, Oh TH, Markelonis GJ, Cho JW, Oh YJ (2004) Phosphorylation of p38 MAPK induced by oxidative stress is linked to activation of both caspase-8- and -9-mediated apoptotic pathways in dopaminergic neurons. J Biol Chem 279:2045120460.

Chung YM, Yoo YD, Park JK, Kim YT, Kim HJ (2001) Increased expression of peroxiredoxin II confers resistance to cisplatin. Anticancer Res 21:1129-1133.

De Simoni S, Goemaere J, Knoops B (2008) Silencing of peroxiredoxin 3 
and peroxiredoxin 5 reveals the role of mitochondrial peroxiredoxins in the protection of human neuroblastoma SH-SY5Y cells toward MPP+. Neurosci Lett 433:219-224.

Ding YM, Jaumotte JD, Signore AP, Zigmond MJ (2004) Effects of 6-hydroxydopamine on primary cultures of substantia nigra: specific damage to dopamine neurons and the impact of glial cell line-derived neurotrophic factor. J Neurochem 89:776-787.

Faigle R, Brederlau A, Elmi M, Arvidsson Y, Hamazaki TS, Uramoto H, Funa K (2004) ASK1 inhibits astroglial development via p38 mitogenactivated protein kinase and promotes neuronal differentiation in adult hippocampus-derived progenitor cells. Mol Cell Biol 24:280-293.

Fang J, Nakamura T, Cho DH, Gu Z, Lipton SA (2007) S-nitrosylation of peroxiredoxin 2 promotes oxidative stress-induced neuronal cell death in Parkinson's disease. Proc Natl Acad Sci U S A 104:18742-18747.

Farrer MJ (2006) Genetics of Parkinson disease: paradigm shifts and future prospects. Nat Rev Genet 7:306-318.

Fujino G, Noguchi T, Matsuzawa A, Yamauchi S, Saitoh M, Takeda K, Ichijo H (2007) Thioredoxin and TRAF family proteins regulate reactive oxygen species-dependent activation of ASK1 through reciprocal modulation of the N-terminal homophilic interaction of ASK1. Mol Cell Biol 27:8152-8163.

Gao Y, Signore AP, Yin W, Cao G, Yin XM, Sun F, Luo Y, Graham SH, Chen J (2005) Neuroprotection against focal ischemic brain injury by inhibition of c-Jun N-terminal kinase and attenuation of the mitochondrial apoptosis-signaling pathway. J Cereb Blood Flow Metab 25:694-712.

Hanrott K, Gudmunsen L, O’Neill MJ, Wonnacott S (2006) 6-hydroxydopamine-induced apoptosis is mediated via extracellular auto-oxidation and caspase 3-dependent activation of protein kinase Cdelta. J Biol Chem 281:5373-5382.

Harada C, Nakamura K, Namekata K, Okumura A, Mitamura Y, Iizuka Y, Kashiwagi K, Yoshida K, Ohno S, Matsuzawa A, Tanaka K, Ichijo H, Harada T (2006) Role of apoptosis signal-regulating kinase 1 in stressinduced neural cell apoptosis in vivo. Am J Pathol 168:261-269.

Jin MH, Lee YH, Kim JM, Sun HN, Moon EY, Shong MH, Kim SU, Lee SH, Lee TH, Yu DY, Lee DS (2005) Characterization of neural cell types expressing peroxiredoxins in mouse brain. Neurosci Lett 381:252-257.

Kadowaki H, Nishitoh H, Urano F, Sadamitsu C, Matsuzawa A, Takeda K, Masutani H, Yodoi J, Urano Y, Nagano T, Ichijo H (2005) Amyloid beta induces neuronal cell death through ROS-mediated ASK1 activation. Cell Death Differ 12:19-24.

Kanayama A, Miyamoto Y (2007) Apoptosis triggered by phagocytosisrelated oxidative stress through FLIPS down-regulation and JNK activation. J Leukoc Biol 82:1344-1352.

Karunakaran S, Diwakar L, Saeed U, Agarwal V, Ramakrishnan S, Iyengar S, Ravindranath V (2007) Activation of apoptosis signal regulating kinase 1 (ASK1) and translocation of death-associated protein, Daxx, in substantia nigra pars compacta in a mouse model of Parkinson's disease: protection by alpha-lipoic acid. FASEB J 21:2226-2236.

Klintworth H, Newhouse K, Li T, Choi WS, Faigle R, Xia Z (2007) Activation of c-Jun $\mathrm{N}$-terminal protein kinase is a common mechanism underlying paraquat- and rotenone-induced dopaminergic cell apoptosis. Toxicol Sci 97:149-162.

Knopman DS, Parisi JE, Salviati A, Floriach-Robert M, Boeve BF, Ivnik RJ, Smith GE, Dickson DW, Johnson KA, Petersen LE, McDonald WC, Braak H, Petersen RC (2003) Neuropathology of cognitively normal elderly. J Neuropathol Exp Neurol 62:1087-1095.

Krapfenbauer K, Engidawork E, Cairns N, Fountoulakis M, Lubec G (2003) Aberrant expression of peroxiredoxin subtypes in neurodegenerative disorders. Brain Res 967:152-160.

Larsen KE, Fon EA, Hastings TG, Edwards RH, Sulzer D (2002) Methamphetamine-induced degeneration of dopaminergic neurons involves autophagy and upregulation of dopamine synthesis. J Neurosci 22: 8951-8960.

Lee YM, Park SH, Shin DI, Hwang JY, Park B, Park YJ, Lee TH, Chae HZ, Jin $\mathrm{BK}, \mathrm{Oh} \mathrm{TH}, \mathrm{Oh} \mathrm{YJ}$ (2008) Oxidative modification of peroxiredoxin is associated with drug-induced apoptotic signaling in experimental models of Parkinson disease. J Biol Chem 283:9986-9998.
Liu H, Zhang H, Iles KE, Rinna A, Merrill G, Yodoi J, Torres M, Forman H) (2006) The ADP-stimulated NADPH oxidase activates the ASK-1/ MKK4/JNK pathway in alveolar macrophages. Free Radic Res 40:865874.

Low FM, Hampton MB, Winterbourn CC (2008) Peroxiredoxin 2 and peroxide metabolism in the erythrocyte. Antioxid Redox Signal 10:16211630.

Niso-Santano M, González-Polo RA, Bravo-San Pedro JM, Gómez-Sánchez R, Lastres-Becker I, Ortiz-Ortiz MA, Soler G, Morán JM, Cuadrado A, Fuentes JM (2010) Activation of apoptosis signal-regulating kinase 1 is a key factor in paraquat-induced cell death: modulation by the Nrf2/Trx axis. Free Radic Biol Med 48:1370-1381.

Ouyang M, Shen X (2006) Critical role of ASK1 in the 6-hydroxydopamineinduced apoptosis in human neuroblastoma SH-SY5Y cells. J Neurochem 97:234-244.

Palacino JJ, Sagi D, Goldberg MS, Krauss S, Motz C, Wacker M, Klose J, Shen J (2004) Mitochondrial dysfunction and oxidative damage in parkindeficient mice. J Biol Chem 279:18614-18622.

Park SH, Chung YM, Lee YS, Kim HJ, Kim JS, Chae HZ, Yoo YD (2000) Antisense of human peroxiredoxin II enhances radiation-induced cell death. Clin Cancer Res 6:4915-4920.

Przedborski S, Ischiropoulos H (2005) Reactive oxygen and nitrogen species: weapons of neuronal destruction in models of Parkinson's disease. Antioxid Redox Signal 7:685-693.

Qu D, Rashidian J, Mount MP, Aleyasin H, Parsanejad M, Lira A, Haque E, Zhang Y, Callaghan S, Daigle M, Rousseaux MW, Slack RS, Albert PR, Vincent I, Woulfe JM, Park DS (2007) Role of Cdk5-mediated phosphorylation of Prx2 in MPTP toxicity and Parkinson's disease. Neuron 55:37-52.

Rhee SG, Chae HZ, Kim K (2005) Peroxiredoxins: a historical overview and speculative preview of novel mechanisms and emerging concepts in cell signaling. Free Radic Biol Med 38:1543-1552.

Saito Y, Nishio K, Ogawa Y, Kinumi T, Yoshida Y, Masuo Y, Niki E (2007) Molecular mechanisms of 6-hydroxydopamine-induced cytotoxicity in PC12 cells: involvement of hydrogen peroxide-dependent and -independent action. Free Radic Biol Med 42:675-685.

Sarafian TA, Verity MA, Vinters HV, Shih CC, Shi L, Ji XD, Dong L, Shau H (1999) Differential expression of peroxiredoxin subtypes in human brain cell types. J Neurosci Res 56:206-212.

Signore AP, Weng Z, Hastings T, Van Laar AD, Liang Q, Lee YJ, Chen J (2006) Erythropoietin protects against 6-hydroxydopamine-induced dopaminergic cell death. J Neurochem 96:428-443.

Stetler RA, Cao G, Gao Y, Zhang F, Wang S, Weng Z, Vosler P, Zhang L, Signore A, Graham SH, Chen J (2008) Hsp27 protects against ischemic brain injury via attenuation of a novel stress-response cascade upstream of mitochondrial cell death signaling. J Neurosci 28:13038-13055.

Takeda K, Matsuzawa A, Nishitoh H, Tobiume K, Kishida S, Ninomiya-Tsuji J, Matsumoto K, Ichijo H (2004) Involvement of ASK1 in Ca2+induced p38 MAP kinase activation. EMBO Rep 5:161-166.

Tsang AH, Chung KK (2009) Oxidative and nitrosative stress in Parkinson's disease. Biochim Biophys Acta 1792:643-650.

Weng Z, Signore AP, Gao Y, Wang S, Zhang F, Hastings T, Yin XM, Chen J (2007) Leptin protects against 6-hydroxydopamine-induced dopaminergic cell death via mitogen-activated protein kinase signaling. J Biol Chem 282:34479-34491.

Yang CS, Shin DM, Lee HM, Son JW, Lee SJ, Akira S, Gougerot-Pocidalo MA, El-Benna J, Ichijo H, Jo EK (2008) ASK1-p38 MAPK-p47phox activation is essential for inflammatory responses during tuberculosis via TLR2ROS signalling. Cell Microbiol 10:741-754.

Zhao W, Fan GC, Zhang ZG, Bandyopadhyay A, Zhou X, Kranias EG (2009) Protection of peroxiredoxin II on oxidative stress-induced cardiomyocyte death and apoptosis. Basic Res Cardiol 104:377-389.

Zhu JH, Kulich SM, Oury TD, Chu CT (2002) Cytoplasmic aggregates of phosphorylated extracellular signal-regulated protein kinases in Lewy body diseases. Am J Pathol 161:2087-2098. 